\title{
Anwendungen
}

Tizian Schröder* und Christian Diedrich

\section{Systemarchitektur für die Implementierung proaktiver Verwaltungsschalen auf Basis kognitionswissenschaftlicher Konzepte}

\author{
System architecture for the implementation of proactive administration shells based on \\ cognitive science concepts
}

https://doi.org/10.1515/auto-2019-0030

Empfangen 21. Februar 2019; angenommen 24. Mai 2019

Zusammenfassung: Der Digitalisierungsprozess hat durch die Initiative „Industrie 4.0“ an Bedeutung gewonnen. Eines der Kernkonzepte ist die Erstellung eines informationstechnischen Repräsentanten von Assets. Für diesen informationstechnischen Repräsentanten gibt es verschiedene Namen am Markt. Der Begriff der Verwaltungsschale wurde dafür durch die Plattform Industrie 4.0 geprägt. In den vergangen Jahren sind Dokumente und Aktivitäten um die Verwaltungsschale entstanden, die entweder Anforderungsbeschreibungen darstellen oder hinsichtlich ihres konkret umsetzbaren Gehaltes keine genügende Präzision haben. Der Beitrag leitet ausgehend vom Systembegriff und kognitionswissenschaftlichen Grundlagen die Systemarchitektur proaktiver Verwaltungsschalen ab. Diese Architektur zeigt auf, wie Verwaltungsschalen arbeiten müssten, um tatsächlich das Ziel der Industrie 4.0 verwirklichen zu können.

Schlagwörter: Industrie 4.0, Kognitionswissenschaften, Verwaltungsschale, Systemarchitektur, Proaktivität, Optimierung

Abstract: The digitisation process has gained importance through the "Industrie 4.0" initiative. One of the core concepts is the creation of a digital representative of assets. There are various names on the market for such a digital representative. The term "administration shell" was coined by the "Industrie 4.0" platform. In the past years documents and activities arose around the administration shell, which either represent requirement descriptions or specifications which lack sufficient precision in order to actually implement them. This paper derives the system

*Korrespondenzautor: Tizian Schröder, OVGU Magdeburg, Magdeburg, Deutschland, E-Mail: tizian.schroeder@ovgu.de Christian Diedrich, OVGU Magdeburg, Magdeburg, Deutschland, E-Mail: christian.diedrich@ovgu.de architecture of proactive administration shells from the term of "systems" and cognitive science concepts. This architecture shows how administration shells would have to work in order to meet the goals of "Industrie 4.0".

Keywords: Industrie 4.0, cognitive sciences, administration shell, system architecture, proactivity, optimization

\section{Hintergrund}

Ein zentraler Begriff dieser Arbeit ist der der Verwaltungsschale (engl.: Asset Administration Shell, kurz: AAS) [1]. Dieser wurde durch die Plattform Industrie 4.0 geprägt, in der sich bedeutende deutsche Industrieverbände zusammengeschlossen haben, um Konzepte und Technologien für die Umsetzung des industriellen Internets der Dinge (IIoT) zu erarbeiten. Diese „Dinge“ werden im Umfeld der Diskussionen um Industrie 4.0 (kurz: 14.0) auch als Assets bezeichnet. Der Idee der cyber-physikalischen (Produktions-)Systeme ( $\mathrm{CP}(\mathrm{P}) \mathrm{S})$ folgend, werden diese Assets durch eine digitale Repräsentanz erweitert, um eine Integration der physischen Welt in die Informationswelt vorzunehmen (Digitalisierung). Für diesen informationstechnischen Repräsentanten gibt es verschiedene alternative Bezeichnungen am Markt, wie z. B. „digitaler Zwilling“. Diese Bezeichnungen werden je nach Sichtweise als synonym oder mit abweichender Bedeutung besetzt aufgefasst. In dieser Arbeit werden diese als Synonyme angesehen, da die verbreiteten Differenzierungen im Wesentlichen auf einen graduellen Unterschied bezüglich des Umfangs der durch diese Repräsentanten vorgehaltenen Daten und Fähigkeiten abstellen. Es ist fraglich, ob diese graduellen Unterschiede begriffsprägend sind.

Abbildung 1 verdeutlicht den angedachten Zweck einer AAS als solch eine digitale Repräsentanz.

Eine AAS verfügt über zwei Schnittstellen: eine grundsätzlich proprietäre Schnittstelle zur Kommunika- 


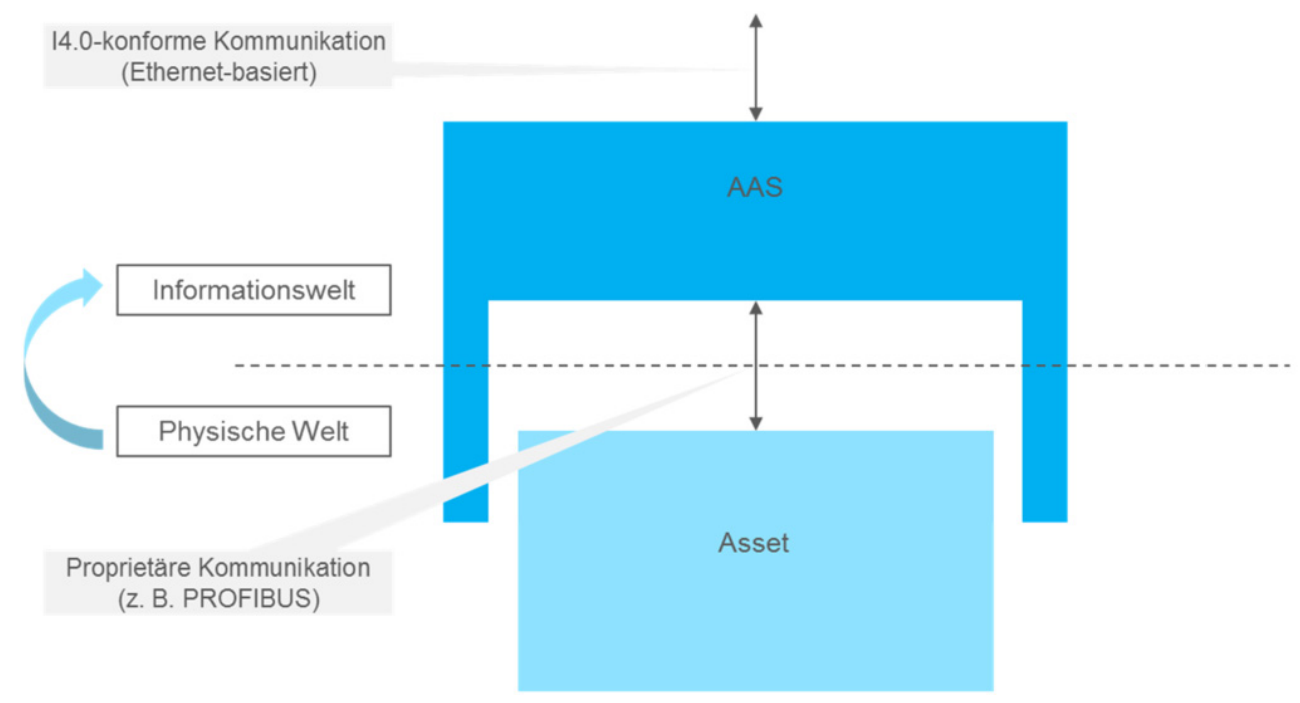

Abb. 1: Verwaltungsschale (AAS).

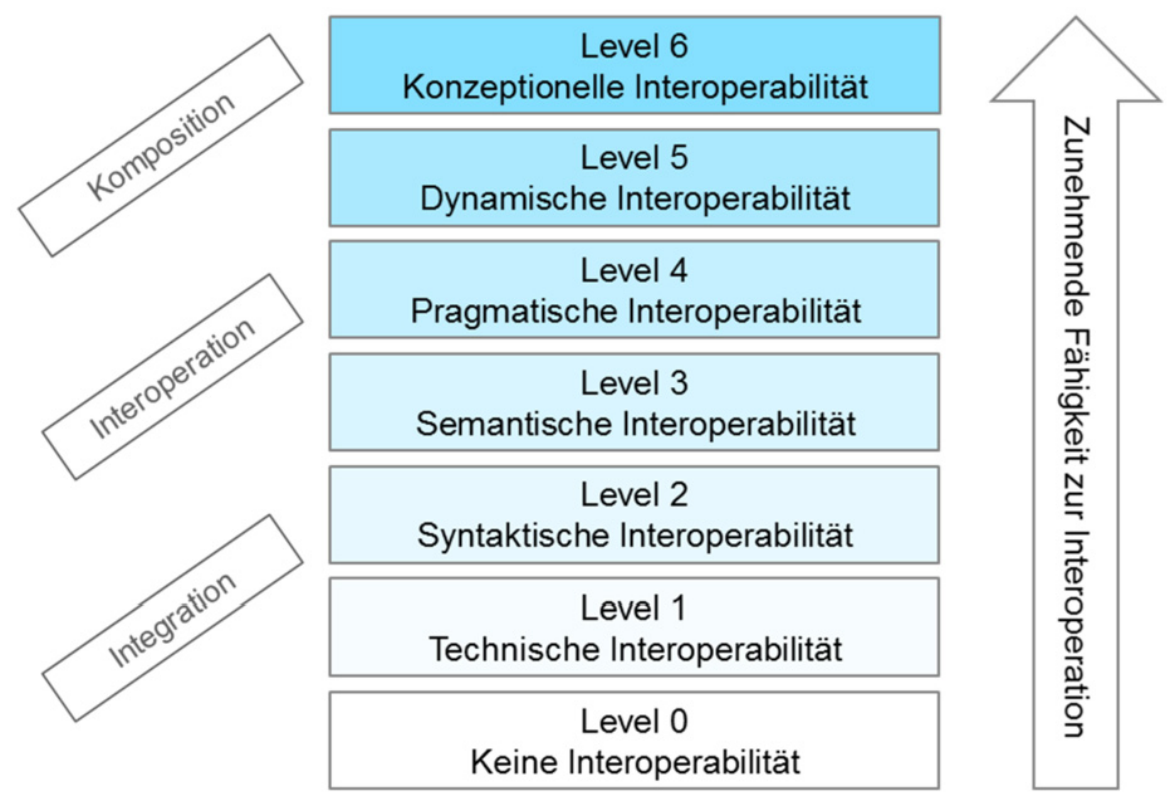

Abb. 2: Levels of Conceptual Interoperability Model (LCIM).

tion zwischen AAS und Asset sowie eine I4.0-konforme Schnittstelle zur Kommunikation von AASs untereinander. „I4.0-konform“ bedeutet hierbei zunächst lediglich, dass durch diese Konformität technische und syntaktische Interoperabilität zwischen AASs eines aus mehreren AASs zusammengesetzten 14.0-Systems gegebenen ist. Zur Einordnung dieser Anforderungen dient Abbildung 2, die das Levels of Conceptual Interoperability Model (LCIM) als ein gängiges Modell zu den Stufen von Interoperabilität veranschaulicht [2]. Höhere Stufen der Interoperabilität werden im Verlauf dieser Arbeit adressiert, wobei jedoch auf- grund konzeptioneller Schwächen dieses Modells nicht alle Stufen des LCIM einbezogen werden.

Die Vision der I4.0 sieht nun vor, dass eine Vielzahl von AASs ein dezentral organisiertes I4.0-System bildet, in dem autonome, intelligente Systemteilnehmer zum Zweck ihrer individuellen Zielerreichung agieren. Individualität kann naheliegender Weise Kooperation erfordern, sodass hierdurch kein Egoismus impliziert wird. Als grundlegendste Motivation dieser Bemühung kann es angesehen werden, die schon heute enorme und zukünftig potentiell noch weiter wachsende Komplexität von Systemen da- 


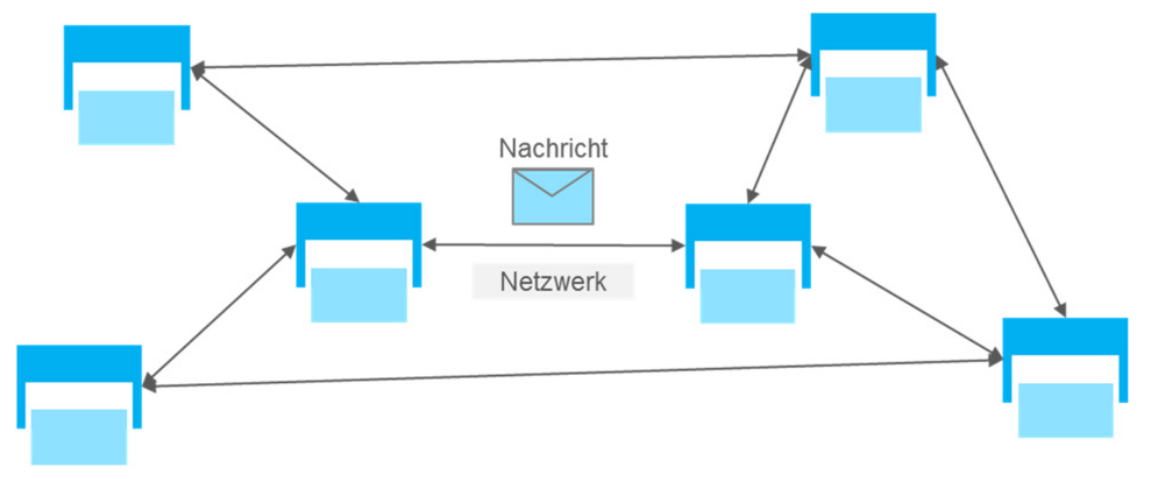

Abb. 3: 14.0-System mit nachrichtenbasierter Interaktion.

durch besser oder überhaupt erst beherrschbar zu machen, dass von einem zentralistischen zu einem dezentralen Ansatz übergegangen wird. Abbildung 3 zeigt schematisch ein einfaches I4.0-System, in dem dezentrale Akteure nachrichtenbasiert interagieren.

Das Interaktionsverhalten einer AAS ist in dem hier betrachteten allgemeinen Fall charakterisiert durch:

- Asynchronität

- Eine AAS wird nicht durch eine eingegangene Interaktion in ihrem übrigen Verhalten blockiert.

- Nicht-Determinismus

- Externe AASs (Empfänger) verhalten sich aus Sicht der betrachteten AAS (Sender) nichtdeterministisch.

- Zustandsbehaftung

- Das Verhalten einer AAS ist zustandsabhängig.

Es sind natürlich Systeme denkbar, in denen sich die Akteure (hier: AASs) hinsichtlich ihres Interaktionsverhalten anders darstellen. Das komplexeste, aber auch das größtmögliche Maß an Autonomie ermöglichende Interaktionsverhalten, ist aber das zuvor vorgestellte. Dieses soll im Rahmen dieser Arbeit zugrunde gelegt werden, da diesbezüglich der größte Forschungsbedarf besteht. Systeme mit alternativen Interaktionscharakteristika wie SOA oder REST sind hinreichend betrachtet und unter Hinnahme derer Schwächen (siehe hierzu [3]) auch angewendet worden.

Folgt man den ersten abstrakten Festlegungen zum Zweck einer AAS [4], so lässt sich leicht folgern, dass eine AAS stets ein aktives Gebilde ist. Eine AAS zeigt im Allgemeinen sowohl reaktives als auch proaktives Verhalten als Ausprägungsformen von aktivem Verhalten. Reaktiv meint hierbei das Reagieren auf Interaktionsanfragen externer Systemteilnehmer. Proaktiv meint das Initiieren von aktivem Verhalten und ggf. an externe Systemteilnehmer gerichteter Anfragen durch eine AAS selbst. Die bis- herigen lösungsbezogenen $-\mathrm{d}$. h. nicht lediglich anforderungsdefinierenden - Festlegungen der Plattform Industrie 4.0 adressieren ausschließlich das reaktive Verhalten von AASs und selbst diesbezüglich nur den kleinen Teilaspekt der gegenseitigen Informationsabfrage. Ein wesentlicher Beitrag der in dieser Arbeit vorgestellten Systemarchitektur ist die Erweiterung der Ideen zur AAS um proaktive Bestandteile.

Wie es für die Ingenieurswissenschaften üblich ist, soll sich die angestrebte Systemarchitektur aus bestehenden, grundlagenorientierten Wissenschaften ableiten lassen. Im Umfeld der I4.0 fallen Begriffe wie „Intelligenz“, „Autonomie“ oder „Dezentralität“ [4]. Ein Wissenschaftskomplex, der diese Begriffe intensiv zum Forschungsgegenstand hat, sind die Kognitionswissenschaften. Dies ist ein interdisziplinäres Gebiet, bestehend aus Inhalten der Philosophie, Psychologie, Neurowissenschaft, Linguistik und ausgewählten Aspekten der Informatik. In Debatten unter „Technikern“ wird dem genauen Gehalt solcher Begriffe oft nicht viel Bedeutung beigemessen. Es ist auch nicht Aufgabe von Ingenieuren und Informatikern, diese Begriffe zu definieren, wohl aber die Erkenntnisse der Kognitionswissenschaften bezüglich „Intelligenz“ und „Autonomie“ technisch nutzbar zu machen. Während die Kognitionswissenschaften ein für viele denkbare technische Verbesserungen hinreichendes Verständnis dieser Begriffe bereits liefern, mangelt es dort aber oft an der nötigen Formalität, um dieses Potential technisch nutzbar machen $\mathrm{zu}$ können. Die vorliegende Arbeit soll einen Beitrag zu dessen Nutzbarkeit leisten. Zu erwähnen ist, dass in verschiedenen Kontexten unterschiedliche Begriffsdefinitionen vorliegen. Ein Überblick über verbreitete Lehrmeinungen findet sich in [34]. Gemein ist diesen Sichtweisen auf den Begriff der Intelligenz, dass es sich hierbei um eine eigenständige Erkenntnis- und Entscheidungsfähigkeit handelt, die Akteure in die Lage versetzt, selbständig Probleme zu lösen. Die Problemlösung erfolgt hierbei grundsätz- 
lich autonom (autos=selbst, nomos=Gesetz), d. h. vor dem Hintergrund selbst gesetzter Ziele und unter Einsatz eigener Fähigkeiten. Es kann Teil der eigenständigen Problemlösung sein, selbst nach geeigneten Kooperationspartnern zu suchen und deren Fähigkeiten in Anspruch zu nehmen.

Die im Folgenden vorgestellte Systemarchitektur und die daraus abgeleiteten Implikationen erheben nicht den Anspruch, AASs „einfach“ implementierbar zu machen. Vielmehr soll die Systemarchitektur aufzeigen, wie AASs arbeiten müssten, um tatsächlich die Vision der I4.0 von „intelligenten“, „autonomen“, „dezentral organisierten“ Systemen verwirklichen zu können. Sie ermöglicht auch die Einordnung bisheriger Arbeiten der Gremien der Plattform Industrie 4.0 sowie aktueller Herausforderungen bei der Umsetzung von Industrie 4.0-Szenarien. Im Anschluss an die Vorstellung des generischen Konzepts wird ein Ausblick auf Implementierungsmöglichkeiten mithilfe konkreter Technologien gegeben. Außerdem erfolgt eine Einordnung des Beitrags von konsensualen Verhaltensregeln zur Dezentralisierung von Systemen.

Ziele sind:

- Konzeptioneller Entwurf einer Systemarchitektur für proaktive AASs

Anforderungen:

- Abgeleitet aus kognitionswissenschaftlichen Konzepten

- Allgemeingültig, d. h. keine sinnvolle Implementierung eines I4.0-Szenarios kann der Architektur widersprechen. Dies bedeutet auch, dass ein maximaler Grad an Autonomie [5] damit umsetzbar ist

- Ermöglicht systematische Spezifikationen und Implementierung von AASs, sodass (Design)-Entscheidungen auf fundierten Konzepten statt Ad Hoc-Annahmen aufbauen

- Anwendbar sein für „offene Umgebungen” [6], d.h., Einsatzumgebungen von AASs, die durch die Eigenschaften „unzugänglich“, „nichtdeterministisch“ und „dynamisch“ gekennzeichnet sind

- Einordnung der bisherigen Arbeiten der Plattform I4.0-Gremien

- Einordnung des Beitrags von konsensualen Verhaltensregeln zur Dezentralisierung von Systemen

- Exemplarische Empfehlung von Realisierungstechnologien zur Umsetzung der Konzepte

Die zugrunde liegenden kognitionswissenschaftlichen Konzepte sind:

- Motivationstheorie [7, 8]

- Theorie des rationalen Handelns [9]

\author{
- Phasen des Problemlösens [10] \\ - Modell zum Gedächtnis [11] \\ - Lerntheorien [12]
}

Im Folgenden wird vor allem auf das Modell der Phasen des Problemlösens aus der Psychologie eingegangen, um die Bestandteile der Systemarchitektur und deren Funktionsweise zu begründen. Die übrigen verwendeten Konzepte werden an relevanten Stellen im Text kurz eingeordnet. Um einen detaillierteren Einblick zu ermöglichen, wird auf Literaturstellen verwiesen.

\section{Systembegriff}

Der Begriff des „Systems“ ist in dieser Arbeit entscheidend. Daher soll dieser zunächst formal definiert werden. Die Definition ist eine für die hier verfolgten Zwecke hinsichtlich der Notation angepasste Version der in der Systemtheorie üblichen Definition eines Systems (zu finden bspw. in [13]). Es gilt die Vereinbarung, dass i. Allg. alle Variablen Vektoren sein können.

Ein beliebiges System lässt sich beschreiben durch ein Tupel Sys $=\left(T, X, U, Y, x_{0}, f\right)$ mit:

- Zeitstruktur $T=(T$, succ $) \mathrm{mit}$

- Nachfolgerfunktion succ $: T \rightarrow T, t^{\prime}=\operatorname{succ}(t)$

- Zustandsmenge $X$

- $\quad$ Menge der Eingabezeichen/Input $U$

- Menge der Ausgabezeichen/Output $Y$

- Initialer Zustand $x_{0}$

- Systemfunktion $f=\left(f_{\text {int }}, f_{\text {ext }}\right)$ mit

- $\quad f: X x U \rightarrow X x Y$ mit $\left(x\left(t^{\prime}\right), y\left(t^{\prime}\right)\right)=f(x(t), u(t))$

- $f_{\text {int }}: X x U \rightarrow X$ mit $x\left(t^{\prime}\right)=f_{\text {int }}(x(t), u(t))$

- $f_{\text {ext }}: X x U \rightarrow Y$ mit $y\left(t^{\prime}\right)=f_{\text {ext }}(x(t), u(t))$

Für die später vorgenommenen Betrachtungen zur Formalisierung des Semantikbegriffs wird noch eingeführt, dass sich der Input-/Outputvektor zusammensetzt aus dem für die Semantikzuordnung zu betrachtenden Input/Output und dem restlichen Input/Output:

- $u=\left(u_{\text {cons }}, u_{\text {rest }}\right)$

$-\quad y=\left(y_{\text {cons }}, y_{\text {rest }}\right)$

Systemarchitektur für proaktive AASs

Architekturen für Softwaresysteme - eine AAS wird letztlich eine auf einer Hardware laufende Softwarekomponente sein - gibt es in einer großen Vielzahl. Das gilt auch dann noch, wenn man an solche Softwaresysteme I4.0-typische Anforderungen stellt wie ,autonom“, „kognitiv“ und Ähnliches. Zwei in diesem Umfeld bekannte und vielversprechende Ansätze sind Expertensysteme und 


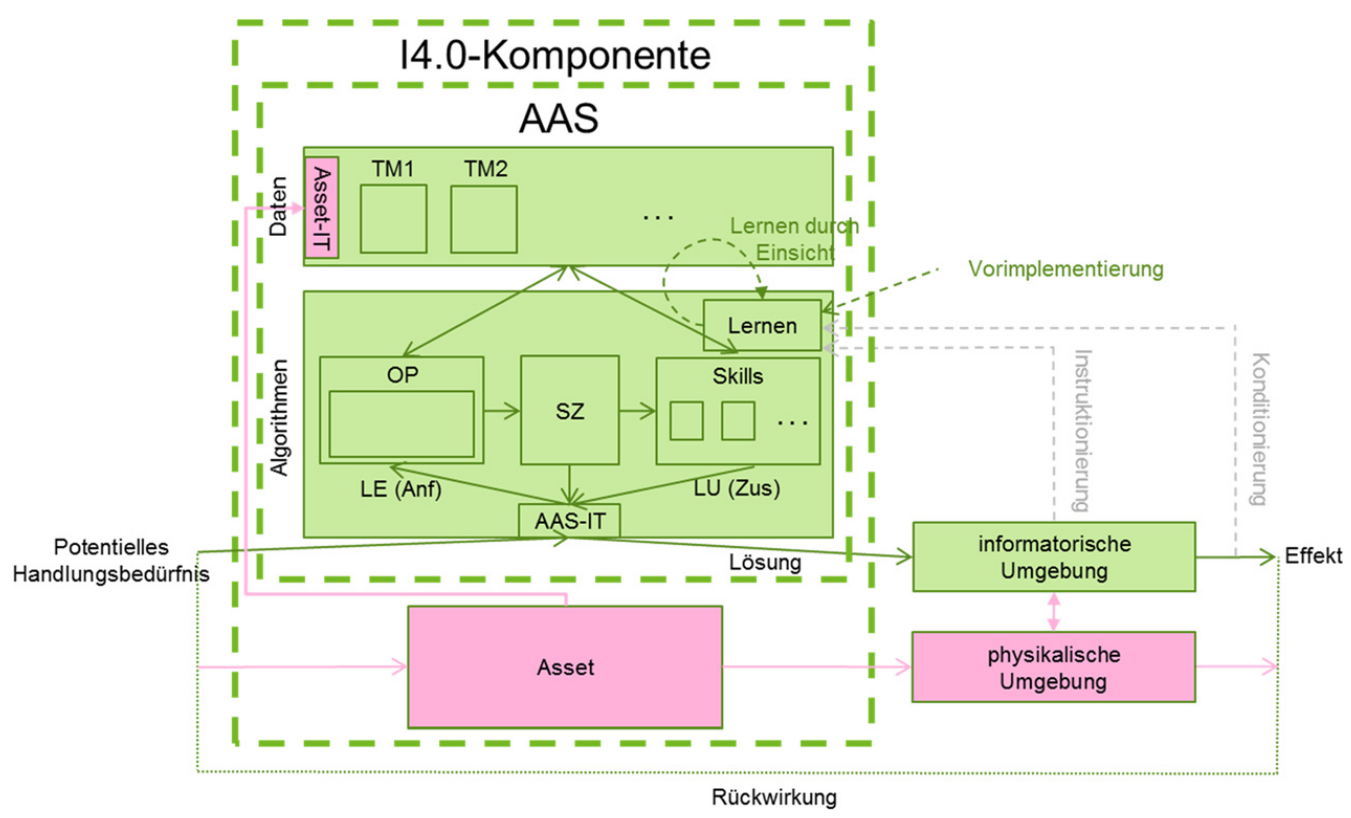

Abb. 4: Systemarchitektur für proaktive AASs.

Agenten. Für den Grobentwurf der neuen Systemarchitektur für proaktive AASs wurden diese beiden Architekturen daher wie später beschrieben zunächst betrachtet und eine Auswahl relevanter Komponenten extrahiert. Diese wurden zur in Abbildung 4 dargestellten neuen Systemarchitektur zusammengeführt. Die genaue Funktionsweise dieser Komponenten und deren (logische) Verbindungen wurden im Einklang mit den einschlägigen kognitionswissenschaftlichen Konzepten definiert.

Die Terminologie der Plattform Industrie 4.0 sieht vor, die Einheit aus AAS und Asset als I4.0-Komponente zu bezeichnen. Eine AAS setzt sich gemäß der vorgeschlagenen Systemarchitektur aus den folgenden funktionalen Komponenten zusammen:

- Daten

- Daten in Teilmodellen (TM) strukturiert

- Algorithmen ${ }^{1}$ mit Sub-Komponenten

- LE - Lösungsermittlung (Anforderungen) durch Lösung eines Optimierungsproblems (OP)

- SZ - Skill-Zuordnung

- Ein Skill bezeichnet eine bestimmte Fähigkeit „etwas zu tun“. Das tatsächliche „Tun“ wird dann als Ausführung des Skills bezeichnet.

- $\quad$ LU - Lösungsumsetzung (Zusicherungen)

- Lernen

- AAS-IT - AAS-Interaktionstreiber

- Asset-IT - Asset-Interaktionstreiber

1 Im I4.0-Umfeld gelegentlich als Component Manager bezeichnet.
Zudem gibt es den Asset-Interaktionstreiber als Schnittstelle zwischen AAS und Asset, der hier nicht weiter betrachtet wird. Dies liegt daran, dass ausdrücklich zwischen physischer und informatorischer Welt unterschieden wird und der Fokus der Betrachtungen auf der Informationswelt liegt. Die physische Welt wird als in der Informationswelt repräsentiert angesehen, sodass derselbe Betrachtungsgegenstand nicht zweimal behandelt werden muss. Zudem besteht der drängendste Forschungsbedarf bezüglich Aspekten, die in der Informationswelt angesiedelt sind. Die Modellierung und Steuerung/Regelung rein physischer Prozesse ist nach dem Stand der Technik, von spezielleren Fragestellungen abgesehen, problemlos möglich. Entsprechend dieser Zweiteilung ist auch die Umgebung, in welche die AAS eingebettet ist und mit der diese in Interaktion tritt, unterteilt. Im Weiteren werden also die rot dargestellten Elemente, d. h. das Asset selbst, dessen Interaktion mit der AAS sowie die physikalische Umgebung grundsätzlich nicht weiter betrachtet.

Die Abbildung veranschaulicht die grobe Funktionsweise einer AAS nach dieser Systemarchitektur: Als Reaktion auf ein über den AAS-IT erfasstes potentielles Handlungsbedürfnis errechnet die Algorithmen-Komponente in 3 prinzipiellen Schritten - der Lösungsermittlung, der Skill-Zuordnung und der Lösungsumsetzung - eine Lösung, welche wiederum über den AAS-IT auf die informatorische Umgebung einwirkt und dort einen Effekt nach sich zieht, der abermals ein potentielles Handlungsbedürfnis für die AAS darstellt. Die Daten-Komponente ent- 

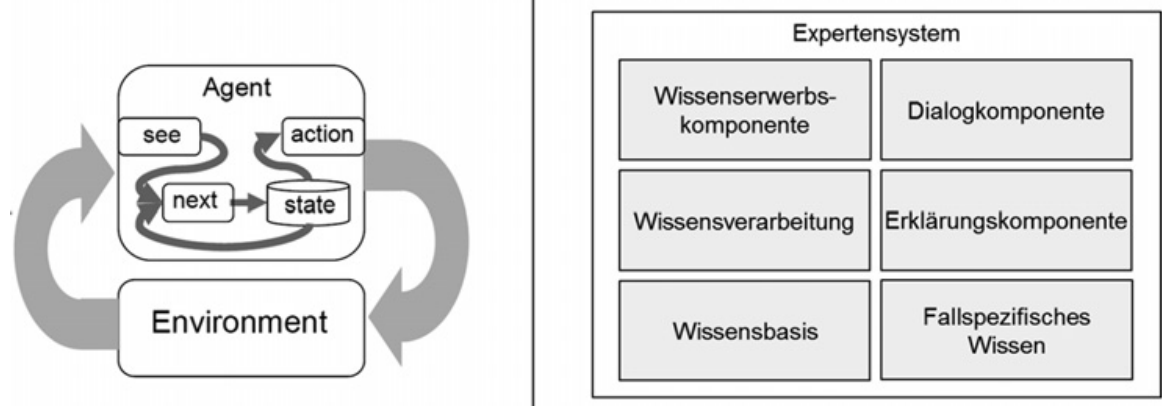

Abb. 5: Grob-Architektur Agent (links, [6]) und Expertensystem (rechts. [17]).

hält deklaratives und prozedurales Wissen, durch das das später vorgestellte generische Modell der Verarbeitung parametriert wird. Die Strukturierung der DatenKomponente entlang dieser Kategorien von Wissen entspricht gängigen Modellen zum Aufbau des Gedächtnisses beim Menschen. Die Anpassung der Daten selbst erfolgt über die Lern-Komponente. Die Form des hier enthaltenen Wissens hängt vom gewählten Ansatz zur Wissensrepräsentation ab. In der I4.0-Debatte werden zurzeit Merkmalmodelle $[1,14]$ favorisiert. Jedoch werden vereinzelt bereits höherwertige Formen wie bspw. Ontologien $[1,15]$ betrachtet. Der später eingeführte Modellierungsvorschlag für proaktives Verhalten von AASs lässt sich zu verschiedenen Repräsentationsformen ausspezialisieren. Um Konformität zum aktuellen Stand der I4.0-Debatte herzustellen, wird angestrebt, dem Metamodellvorschlag der Arbeitsgruppe „Verwaltungsschale im Detail“ [14] zu folgen. Für die Arbeit mit dem in der Daten-Komponente enthaltenen Datenmodell wird das etablierte CRUD-Konzept [16] vorgeschlagen. Dies bedeutet, dass die durch den AASIT bereitgestellte Interaktions-Schnittstelle lediglich die 4 Basisdienste Create, Read, Update sowie Delete zum Operieren auf Elementen des Datenmodells anzubieten braucht. Der konkrete Inhalt der jeweiligen Interaktion hängt dann vom Inhalt der mit diesen Basisdienten behandelten Datenelemente ab. Sämtliche von der Umgebung erfassten Eingaben werden also zunächst - und möglicherweise nur temporär - per Create-Dienst im Datenmodell angelegt, sodass die datenverarbeitenden Algorithmen darauf Zugriff haben.

Abbildung 5 zeigt gängige grobe Architekturen von Agenten [6] und Expertensystemen [17]. Die Komponenten eines Agenten finden sich prinzipiell in der vorgestellten AAS-Systemarchitektur wieder: „see“ geht in der Funktionalität des „Interaktionstreibers“ auf, über den aus der Umwelt stammende Eingaben aufgenommen werden. „next“ entspricht der Ermittlung des nächsten an- zustrebenden Zustands und entspricht somit der „Lösungsermittlung“. Die Zustandshaltung selbst in "state“ erfolgt in der AAS-Systemarchitektur innerhalb der DatenKomponente. Die letztliche nötige Handlung zur Erreichung des nächsten Agenten-Zustandes durch „action“ entspricht der „Lösungsumsetzung“. Jedoch unterscheiden sich die Beziehungen zwischen diesen Komponenten sowie deren interne Funktionsweise teilweise deutlich von der erwähnten Agentenarchitektur. Die Funktionsweise proaktiver AASs beruht dem Vorschlag zufolge auf den in den Kognitionswissenschaften etablierten Phasen des Problemlösens. Es erfolgt demnach ein sequentieller Ablauf von Lösungsermittlung und Lösungsumsetzung, die durch die separate Komponente für die Skill-Zuordnung miteinander verknüpft sind. Somit lässt sich abhängig von der Verfügbarkeit lokaler oder globaler Skills ermitteln, wann und warum überhaupt ein Interaktionsbedarf zwischen autonomen Akteuren wie AASs oder Agenten entsteht. Außerdem wird für den Datenbereich eine spezifische Struktur des darin enthaltenen Datenmodells angestrebt [14]. Weiterhin ist das Konzept für die Modellierung von Verhalten proaktiver AASs darauf ausgelegt, dass AASs gleichzeitig in Interaktion mit mehreren potentiell nicht-deterministisch agierenden AASs treten und somit eine Koordination der daraus möglicherweise resultierenden Zielkonflikte vornehmen müssen (Abbildung 7, Gleichung 1). Die Berücksichtigung dieses im Allgemeinen vorliegenden Nicht-Determinismus erhöht die Flexibilität der so konzipierten und implementierten AASs.

Genauso lassen sich auch die Komponenten eines Expertensystems abbilden: Die „Wissenserwerbskomponente“ entspricht der Komponente „Lernen“. Die gesamte „Wissensverarbeitung“ geht in der AlgorithmenKomponente auf. Die „Wissensbasis“ entspricht der „Daten-Komponente“. Darin geht auch die Komponente „Fallspezifisches Wissen“ auf. Dies bedeutet, dass in der AAS-Systemarchitektur nicht verschiedene Komponen- 
ten für verschiedene Kategorien von Wissen eingeführt werden. Stattdessen finden sich diese Kategorien innerhalb der Daten-Komponente innerhalb eines entlang der verschiedenen Wissenskategorien strukturierten Informationsmodells. Ebenso wird nicht zwischen mehreren Schnittstellen für verschiedene Zwecke der Interaktion unterschieden. Eine AAS besitzt den Interaktions-Treiber über den sämtliche Interaktion abgewickelt wird: Dialoge, Erklärungen, Entwicklereingriffe. Natürlich haben diese Interaktionen einen unterschiedlichen inhaltlichen Charakter. Um dies abzubilden, sind jedoch nicht mehrere Interaktionskomponenten nötig. Stattdessen werden in einer AAS innerhalb der Komponente „Lösungsumsetzung “verschiedene Skills angeboten, die den generischen Interaktions-Treiber anweisen, Interaktion mit durch die Skills bestimmten Inhalten auszuführen.

Die Lern-Komponente kann konzeptionell alle etablierten Lerntheorien implementieren. Grundsätzlich müssen Systemteilnehmer etwas übereinander wissen, um sinnvoll in Interaktion treten zu können. Andernfalls ließen sich nur zufällige und daher mit hoher Wahrscheinlichkeit sinnlose Interaktionen vornehmen. Dieses im Gedächtnis hinterlegte Wissen kann durch verschiedene Lernverfahren durch eine AAS erworben werden. Im Folgenden sind die gängigen Kategorien von Lernverfahren ihrer Mächtigkeit nach aufsteigend dargestellt [12, 17].

- kein Lernen:

- (1) Vorimplementierung ${ }^{2}$

- ohne Inferenz:

- (2) Instruktionismus ${ }^{3}$

- (3) (operante) Konditionierung, Lernen am Modell

- mit Inferenz:

- (4) Lernen durch Einsicht, innere Vorgänge ${ }^{4}$

Aus ingenieurwissenschaftlicher Sicht wären ausgehend von diesen Lerntheorien technische Umsetzungen $\mathrm{zu}$ entwickeln. Jedoch liefert die Informatik hier bereits zahlreiche Lösungen, die sich problemlos in die LernKomponente und somit in die Systemarchitektur integrieren ließen [33]. Daher soll die Aufgabe des Entwurfs ei-

2 Hierbei wird Wissen durch den Entwickler manuell in das Gedächtnis eingebracht. Somit handelt es sich hierbei streng genommen nicht um Lernen, jedoch wird dies konzeptionell durchaus zu den (schwachen) Lerntheorien gezählt.

3 Diese Form findet sich aktuell stark in Aktivitäten der Plattform Industrie 4.0 wieder. So sollen z. B. Komponenten ihre Fähigkeiten explizit durch Informationsmodelle für externe Systemteilnehmer erkundbar machen („Selbstbeschreibung“).

4 Hierbei wird Wissen durch den Lernenden aus bereits bestehendem Wissen abgeleitet. ner generischen Gesamtarchitektur nicht unnötig durch Betrachtung höherwertiger Lernverfahren verkompliziert werden. Im Folgenden wird daher der Ansatz der Vorimplementierung des durch die AAS benötigten Wissens durch den Entwickler und teilweise das Lernen durch Einsicht gewählt (in Abbildung 4 grün hervorgehoben). Die konzeptionellen Aussagen, die diese Arbeit liefert, werden durch diese vereinfachende Annahme in keiner Weise abgeschwächt oder eingeschränkt.

\section{Generisches Modell der Verarbeitung}

In der allgemeinen Psychologie sind folgende Phasen des Problemlösens anerkannt [10]:

- Phase 1: Problemidentifikation

- Phase 2: Ziel- und Situationsanalyse

- Phase 2a: Zielanalyse

- Phase 2b: Situationsanalyse

- Phase 3: Planerstellung

- Phase 4: Planausführung

- Phase 5: Evaluation

Abbildung 6 detailliert die bereits umrissene Funktionsweise einer AAS nach dieser Systemarchitektur. Hieran lässt sich auch die direkte Umsetzung des Modells der Phasen der Problemlösung erkennen. Es erfolgt zunächst eine intuitive Erklärung der Funktionsweise bevor später einzelne Aspekte formal dargestellt werden.

Die Phase 1: Problemidentifikation ist bei der hier vorgeschlagenen Funktionsweise keine eigenständige Phase, da diese in der Phase 2b: Situationsanalyse aufgeht. Denn über den AAS-IT wird fortlaufend die Ist-Situation der Umgebung erfasst und es besteht Zugriff auf ZustandsInformation aus der Daten-Komponente. Zugleich ist die handlungsleitende Motivation einer jeden AAS explizit durch eine Zielfunktion - i. Allg. sind multiple Zielfunktionen abbildbar - repräsentiert (Phase 2a: Zielanalyse). Dieser Sachverhalt wird im weiteren Verlauf der Arbeit noch anhand einer abgeleiteten Form für I4.0-spezifische dynamische Optimierungsprobleme (kurz: $\mathrm{OP}$ ) genauer dargelegt. Die Tatsache, dass das durch den Entwickler angedachte Verhalten einer AAS in Form von OPs deklarativ beschrieben wird, macht auch deutlich, weshalb die Problemidentifikation hier nicht mehr als eigenständige Phase betrachtet zu werden braucht: Denn aus dem kontinuierlichen Lösen des das Verhalten einer AAS deklarierenden dynamischen OPs ergibt sich unmittelbar auch die Problemsituation (potentielles Handlungsbedürfnis). 


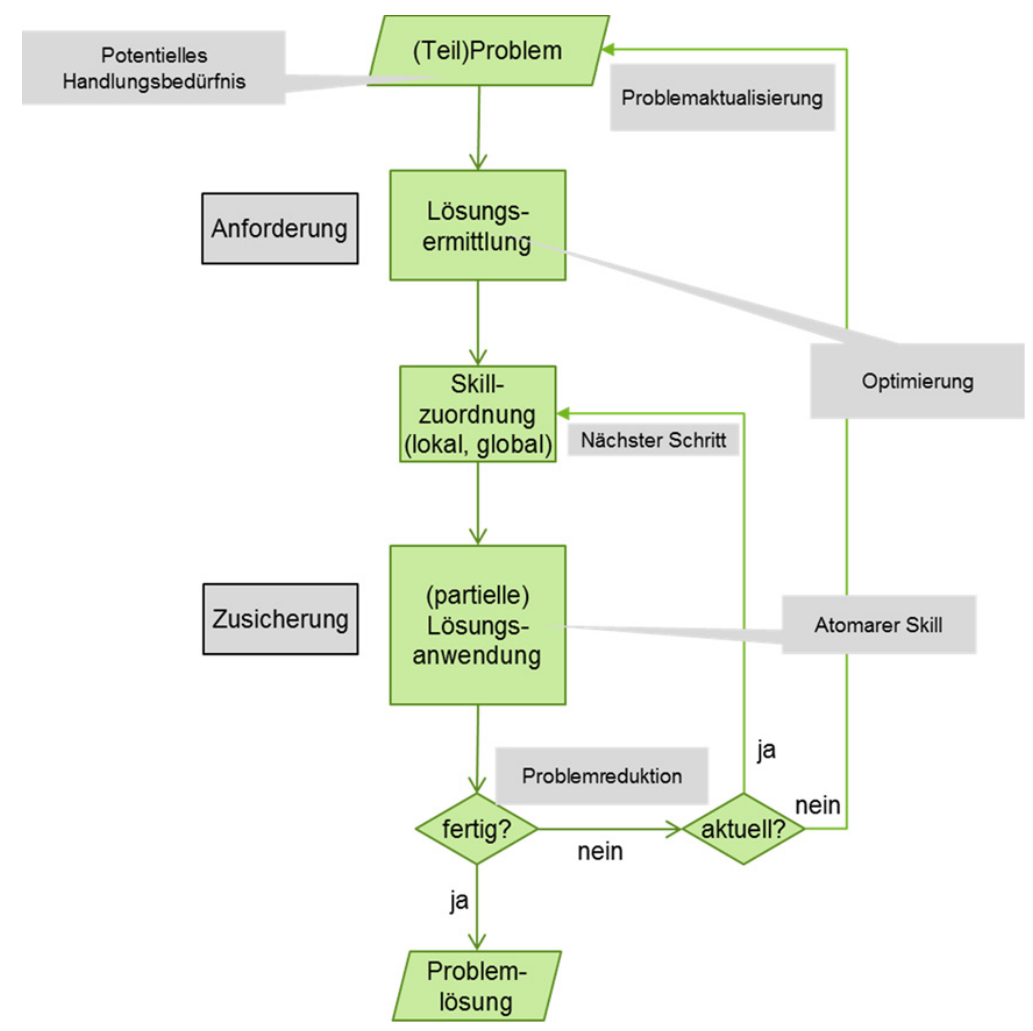

Abb. 6: Funktionsweise einer AAS.

Intuitiv lässt sich das so vorstellen, dass die aktuelle Lösungsermittlung des OPs kein von dem aktuellen Verhalten der AAS abweichendes Verhalten vorsieht sofern dadurch keine Zielfunktionswertverbesserung möglich ist (keine Problemsituation), jedoch sehr wohl eine Verhaltensänderung vorsieht, wenn in der aktuellen Situation eine Zielfunktionswertverbesserung erreicht werden kann (Problemsituation). Mit einfachen Worten: Wenn die Zielfunktionserwartungen bereits erfüllt sind oder prinzipiell keine Verbesserung möglich ist, dann liegt kein Problem vor, auf das zu reagieren wäre. Das durch Fakten aus Umgebung und Daten-Komponente parametrierte OP wird im Rahmen der Lösungsermittlung gelöst. Die Lösung eines OP besteht aus Werten, die die Entscheidungsvariablen annehmen müssen, um eine entsprechende Zielfunktionswerterhöhung zu erreichen. Dies macht deutlich, dass die durch die Lösungsermittlung erhaltene Lösung des OPs zunächst nur eine Anforderung, d. h. einen erstellten Plan zur Verbesserung der aktuellen Situation, darstellt (Phase 3: Planerstellung). Diese Lösung ist also im Rahmen der Lösungsumsetzung noch auszuführen (Phase 4: Planausführung). Zu diesem Zweck wird im Rahmen der SkillZuordnung ermittelt, durch welche atomaren Skills die errechnete Belegung der Entscheidungsvariablen zugesichert werden kann. Als atomaren Skill bezeichnen wir ei- nen kleinsten zuordenbaren Verarbeitungsschritt. Nach Anwendung solch eines Skills ist entweder die vollständige Lösungsermittlung verwirklicht worden (Problemlösung) oder nur ein Teil davon umgesetzt (Problemreduktion). Dies bedeutet, dass das Problem entweder gelöst ist oder im Rahmen einer erneuten Skill-Zuordnung der nächste Schritt ${ }^{5}$ aus der Lösungsermittlung einem passenden Skill zugeordnet wird. Zudem gilt, dass eine einmal bestimmte Lösungsermittlung bei Vorliegen einer geänderten Situationen - d. h. einem geänderten Problem durch eine neu zu erstellende Lösungsermittlung zu ersetzen ist (Problemaktualisierung). Daher wird in definierten Zeitabständen eine Neuberechnung des OPs unter Einbeziehung der aktuellen Umgebungs- und Zustandsinformation vorgenommen. Geänderte Situationen können daraus resultieren, dass die Konsequenzen des eigenen Verhaltens einer AAS aufgrund ungenauer Umgebungsmodelle nicht korrekt prädiziert wurden sowie daraus, dass auch andere Akteure (AASs) auf die Umgebung einwirken. Anschließend an die Lösungsumsetzung könnte sich optional eine Phase 5: Evaluation anschließen, um die Adäquat-

5 Die Entscheidungsvariablen sind Teil eines dynamischen OPs, d. h. sie sind zeitabhängig. Daher kann man hier von aufeinanderfolgenden Schritten der Lösung sprechen. 
heit der Lösungsanwendung zu überprüfen. Wie bereits erwähnt und später genauer dargelegt, wird aber i. Allg. von einem dynamischen OP ausgegangen, d. h. einem OP, das kontinuierlich auf zu definierten Zeitpunkten vorliegende Ist-Situationen angewendet wird. Dieses dynamische, fortlaufende Lösen des OPs erfüllt zugleich die Funktion der Evaluation voriger ermittelter und angewandter Lösungen.

Bezüglich der Skill-Zuordnung ist zwischen lokaler und globaler Zuordnung zu unterscheiden. Jede AAS kann über eigene Skills verfügen (s. Abbildung 4). Ergibt sich im Rahmen der Skill-Zuordnung, dass ein lokaler Skill für einen jeweiligen Schritt der Lösungsumsetzung in Frage kommt, so wird dies so vorgenommen (Prio1 - lokale Zuordnung). Im Allg. wird eine einzelne AAS aber nicht über alle benötigten Skills selbst verfügen. Daraus lassen sich Interaktionsbedürfnisse von AASs ableiten: Kann ein (Teil einer) ermittelten Lösungsanforderung nicht durch die AAS selbst realisiert werden, so werden externe AASs um die Zurverfügungstellung geeigneter Skills gebeten. $\mathrm{Zu}$ diesem Zweck müssen I4.0-Komponenten miteinander über den AAS-IT in Interaktion treten (Prio2 - globale Zuordnung). Hierbei findet die I4.0-Sprache Anwendung [18]. Die Skill-Zuordnung, welche Lösungsermittlung und Lösungsumsetzung miteinander verbindet, ist also der entscheidende Schritt, um Interaktionsbedürfnisse abzuleiten. Dies ist ein Vorteil gegenüber dem zurzeit in der PI4.0 verfolgten Ansatz der semantischen Protokolle [18]. Hierbei sollen notwendige Interaktionen mit an einer Problemlösung beteiligten Rollen direkt durch Zustandsmaschinen spezifiziert werden. Es lässt sich also nicht mehr zur Laufzeit durch eine AAS ermitteln, ob überhaupt ein Interaktionsbedürfnis besteht, stattdessen wird ein solches Bedürfnis ausdrücklich vorgeschrieben oder ausgeschlossen. Das Auffinden passender Skills erfolgt durch „Browsing“ des Informationsmodells (Prio1) oder durch Suche geeignet ausgestatteter externer AASs (Prio2) mithilfe eines „Discovery Servers“ (DS). Alle an einem I4.0-System teilnehmenden AASs können sich unter Beschreibung ihrer angebotenen Skills bei solch einem DS registrieren, so dass andere AAS durch Anfrage an den DS erfahren können, welche Skills nutzbar sind.

Gelegentlich wird angemerkt, dass Entscheidungen in der Realität oft nicht als OP abbildbar sind, da die Akteure nicht über rationale Präferenzen verfügen. Was Präferenzen rational macht, ergibt sich aus den Anforderungen an die so genannte von-Neumann-Morgenstern Nutzenfunktion [19]. Der Zweck der Beschreibung der Systemarchitektur und der Funktionsweise der enthaltenen Bausteine ist aber ein anderer. Ziel ist es nicht, gegebene irrationale Akteure abbildbar zu machen, sondern zum einen diese $\mathrm{zu}$ analysieren und erklärbar zu machen, worin die Irrationalität besteht. Zum anderen und vor allem geht es aber darum, rational agierende Systeme zu synthetisieren. Hierbei ist es erst recht sinnvoll, die Anforderungen an rationales Handeln direkt zum Bestandteil des Konzeptes zu machen und damit die Rationalität der mit Hilfe der vorgeschlagenen Systemarchitektur entworfenen Systeme zu garantieren.

\section{Modellierung von proaktivem Verhalten einer AAS}

Das durch den Entwickler vorgesehene Verhalten einer AAS wird deklarativ in Form von OPs spezifiziert, ${ }^{6}$ da dies der kognitionswissenschaftlichen Theorie der rationalen Entscheidung, der Motivationstheorie und insbesondere dem Modell der Phasen des Problemlösens entspricht. Eingangs wurde dargestellt, dass sich ein beliebiges System - also auch jede AAS - durch ein Tupel Sys = $\left(T, X, U, Y, x_{0}, f\right)$ beschreiben lässt. Zentrales Element ist nun die Systemfunktion f. Dies kann zunächst eine völlig beliebige Funktion sein. Für die Synthese von Systemen lässt aus dieser richtigen aber auch sehr abstrakten Feststellung zunächst nicht viel Hilfreiches ableiten. Daher soll eine spezifischere Gestalt von $f=\left(f_{\text {int }}, f_{\text {ext }}\right)$ für AASs im Folgenden abgeleitet werden. Konkret soll die TeilSystemfunktion $y\left(t^{\prime}\right)=f_{\text {ext }}(x(t), u(t))$ spezialisiert werden. Bezüglich der Teil-Systemfunktion $x\left(t^{\prime}\right)=f_{\text {int }}(x(t), u(t))$ ist keine weitere Spezialisierung nötig, um eine unmittelbare Übereinstimmung mit dem Modell der Phasen des Problemlösens herzustellen und keine Spezialisierung möglich, ohne gleichzeitig die Problemklasse zu verkleinern, auf die der angegebene Vorschlag anwendbar ist.

Ein wichtiges Anliegen der Systemarchitektur war es, den proaktiven Charakter einer AAS zu adressieren. Proaktiv meint, von sich aus die Ausführung von Skills und bei Bedarf auch Interaktionen mit anderen Systemteilnehmern selbständig auszulösen. Abbildung 7 zeigt eine minimale Systemarchitektur bestehend aus einer $A A S_{S}$ ( $\mathrm{S}$ für Self) und der mit dieser in Interaktion stehenden Umgebung. Es hängt von der Definition der Systemgrenzen ab, ob diese Umgebung aus einer, mehreren oder allen übrigen am System teilnehmenden AASs besteht. Zur Vereinfachung wird davon ausgegangen, dass es sich hierbei um

6 Statt „Verhaltensspezifikation“ ließe sich auch der Begriff „Algorithmus“ verwenden. Um die große Nähe der vorgestellten Überlegungen zu den Kognitionswissenschaften zu vermitteln, wird dieser technische Begriff aber bewusst oft nicht verwendet. 


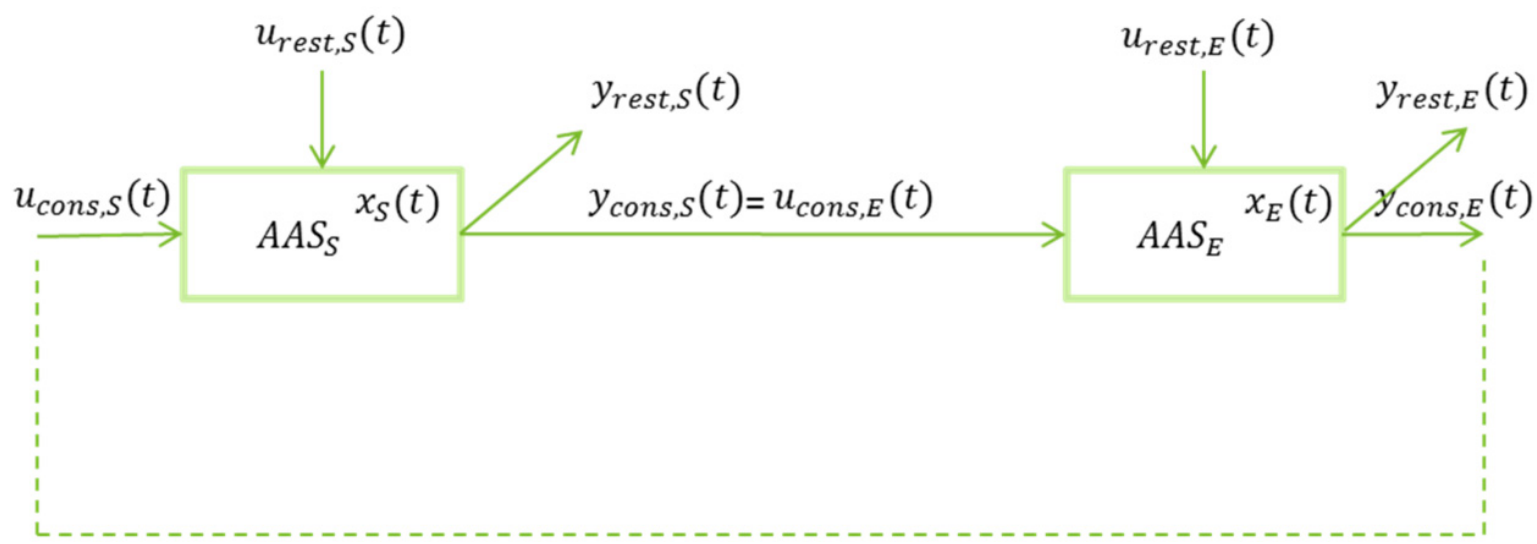

Abb. 7: AAS und ihre Umgebung.

eine beliebig komplexe „Umgebungs-AAS“ $A A S_{E}$ (E für Environment) handelt.

Die Notation für die Beschreibung der Systeme $A A S_{S}$ und $A A S_{E}$ entspricht der eingangs eingeführten allgemeinen Systembeschreibung (Systembegriff) wobei gilt:

- Index S für Self

- eigenes System/betrachtete AAS

- Index E für Environment

- Umgebung der AAS

Die Notation $A A S_{S} \backslash \bigvee_{\text {ext }, S}$ bezeichnet das Tupel $A A S_{S}$ ohne die Komponente $f_{\text {ext }, S}$.

Deutlich wird hierbei nochmal, dass bezüglich der Inputs/Outputs unterschieden wird zwischen den aktuell betrachteten $\left(u_{\text {cons }, S}, y_{\text {cons }, S}, u_{\text {cons }, E}, y_{\text {cons }, E}\right)$ und den restlichen $\left(u_{\text {rest }, S}, y_{\text {rest }, S}, u_{\text {rest }, E}, y_{\text {rest }, E}\right)$. Diese Unterscheidung ist wichtig, da das Wissen der $A A S_{S}$ bezüglich aller Interaktionen von $A A S_{E}$ immer auf die durch $u_{c o n s, E}, y_{c o n s, E}$ repräsentierte Information beschränkt ist. $A A S_{S}$ kennt i. Allg. nicht die Werte von $u_{\text {rest }, E}, y_{\text {rest }, E}$. $A A S_{S}$ kennt jedoch $u_{\text {rest }, S}$, $y_{\text {rest }, S}$, da sie die entsprechenden Interaktionen selbst eingeht. Genau aus dieser Tatsache resultiert auch der bereits oben erwähnte Nicht-Determinismus aller Interaktionspartner. Denn die für $A A S_{S}$ nicht bekannten Interaktionen, die $A A S_{E}$ mit weiteren externen Systemteilnehmern eingeht, führen dazu, dass das konkrete Verhalten von $A A S_{E}$ aus Sicht der $A A S_{S}$ nicht determinierbar ist. Diese Unterscheidung ist an späterer Stelle der Arbeit wichtig und kann für die direkt folgenden Betrachtungen erstmal ausgeblendet ${ }^{7}$ werden.

Die folgende Form wird für die speziellste allgemein definierbare Teil-Systemfunktion $y_{S}\left(t^{\prime}\right)=f_{\text {ext,S }}\left(x_{S}(t), u_{S}(t)\right)$

7 D. h. nicht, dass diese Unterscheidung vernachlässigt wurde. Man muss diese lediglich nicht explizit mitführen. einer jeden AAS vorgeschlagen:

$$
\begin{aligned}
& y_{S}(t)=f_{\text {ext,s }}=\text { solve }(O P) \text {, with } O P: \\
& \max _{y_{s}(t)} \int_{t_{0}}^{t_{F}} F\left(x_{S}(t)\right) d t \\
& \text { s.t.: } A A S_{S} f_{S, e x t}=\left(T_{S}, X_{S}, U_{S}, Y_{S}, x_{0, S}, f_{\text {int }, S}\right) \\
&\left.A A S_{E}=\left(T_{E}, X_{E}, U_{E}, Y_{E}, x_{0, E}, f_{\text {int }, E}, f_{\text {ext }, E}\right)\right)
\end{aligned}
$$

Die Teil-Systemfunktion $f_{\text {ext,S }}$ stellt einen Solver für ein OP dar. Bei diesem OP handelt es sich im Allg. um ein dynamisches, erkennbar an dem integralen Charakter der Zielfunktion. ${ }^{8}$ Die Zielfunktion hängt vom Zustand $x_{S}(t)$ ab, denn die AAS ,interessiert" sich für ihre eigene Situation. Die Annahme, dass diese Zielfunktion zu maximieren sei, schränkt die Allgemeingültigkeit dieser Formulierung nicht ein. Die Entscheidungsvariablen im angegebenen OP sind die Outputs $y_{S}(t)$. Als Nebenbedingungen dieses OPs sind die Eigenheiten sowohl der betrachteten AAS selbst als auch die der Umgebungs-AAS zu berücksichtigen, die sich jeweils aus der zugehörigen Systembeschreibung $A A S_{S} \bigvee_{\text {ext,S }}$ bzw. $A A S_{E}$ ergeben. Bei $A A S_{S} \bigvee_{\text {ext }, S}$ handelt es sich wie oben definiert um die Systembeschreibung von $A A S_{S}$ abzüglich der Teil-Systemfunktion $f_{\text {ext }, S}$. Diese Teil-Systemfunktion ist nicht mehr Teil der Nebenbedingungen, da sie den OP-Solver selbst darstellt. Die übrigen Eigenheiten von $A A S_{S}$ sind aber von dem OP-Solver als Nebenbedingungen zu berücksichtigen, insbesondere die Teil-Systemfunktion $f_{\text {int }, S}$, welche Aufschluss darüber gibt, wie sich der ,interessante“ innere Zustand $x_{S}(t)$ entwickelt. Bei $A A S_{E}$ handelt es sich um das benötigte Modellwissen über die Umgebung. Wie oben erwähnt, müssen Systemteilnehmer etwas übereinander wissen, um sinn-

8 Für den denkbaren Spezialfall eines statischen OPs gilt $t_{0}=t_{F}$. 


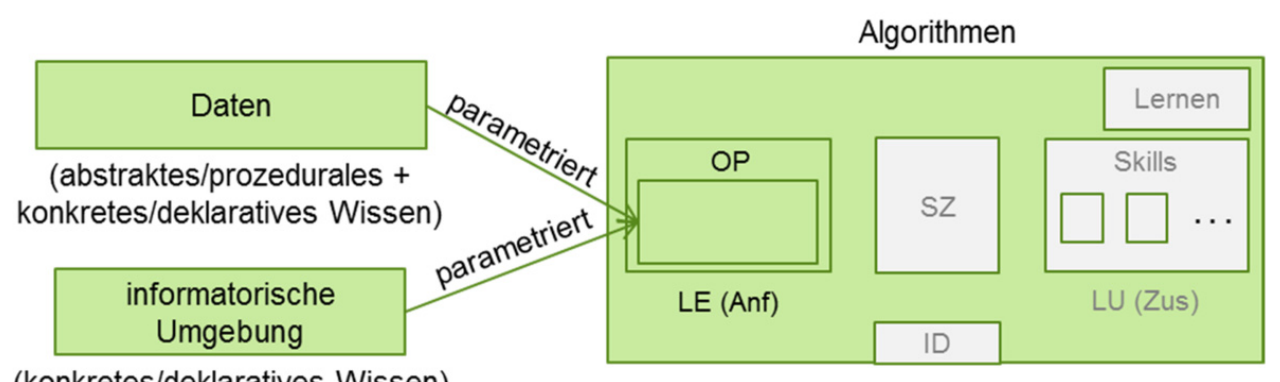

(konkretes/deklaratives Wissen)

Abb. 8: Gedächtnis und Umgebung parametrieren allgemeines OP.

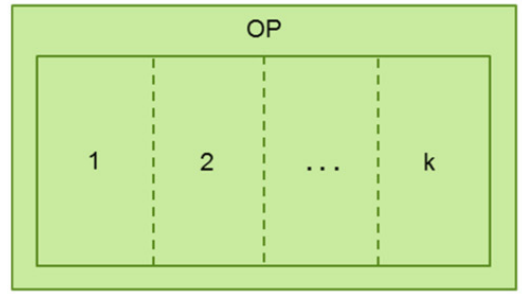

LE (Anf)
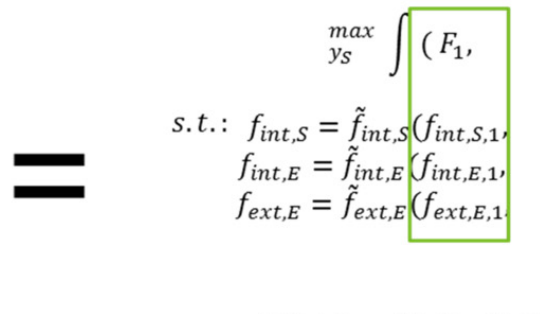

$A A S_{S} \backslash \mathrm{f}_{S}=\left(\mathrm{T}_{\mathrm{S}}, \mathrm{X}_{\mathrm{S}}, \mathrm{U}_{\mathrm{S}}, \mathrm{Y}_{\mathrm{S}}, \mathrm{x}_{0, S}\right)$ $A A S_{E} \backslash \mathrm{f}_{E}=\left(\mathrm{T}_{\mathrm{E}}, \mathrm{X}_{\mathrm{E}}, \mathrm{U}_{\mathrm{E}}, \mathrm{Y}_{\mathrm{E}}, \mathrm{x}_{0, \mathrm{E}}\right)$

Abb. 9: Strukturierung des OPs - Intuition und Formalisierung.

voll in Interaktion treten zu können. Andernfalls ließen sich nur zufällige und daher mit hoher Wahrscheinlichkeit sinnlose Interaktionen vornehmen. Diese allgemeine Form der Systemfunktion wird für konkrete AASs und konkrete Zeitpunkte durch Information aus der DatenKomponente und der Umgebung der AAS parametriert, wie in Abbildung 8 veranschaulicht.

Diese Parametrierung ist die Anreicherung des OPs mit aktuellem Wissen über:

- Ziele

- Quelle: Daten-Komponente

- Entsprechung in OP: Zielfunktion

- Skills

- Quelle: Daten-Komponente

- Entsprechung in OP: setzbare Entscheidungsvariablen (lokal oder global suchen)

- Umgebungsverhalten + eigenes passives Verhalten ${ }^{9}$

- Quelle: Daten-Komponente (prozedural, deklarativ) + Umgebung (deklarativ)

- Entsprechung in OP: Modellwissen/Nebenbedingungen

Die Tatsache, dass hier formal eine Zielfunktion vorgesehen ist, verbietet nicht, dass in dieser mehrere Ziele reprä-

9 Das eigene passive Verhalten einer AAS ist gegeben $\operatorname{durch} f_{\text {int }, S}$. sentiert sein können, eine AAS also mehr als nur ein Ziel verfolgen kann:

$$
F\left(x_{S}(t)\right)=\left(F_{1}\left(x_{S}(t)\right), \ldots, F_{k}\left(x_{S}(t)\right)\right)
$$

Es gibt zahlreiche Methoden zur Lösung von OPs mit mehreren Zielfunktionen [20]. Der Ansatz des Scalarizings bspw. sieht einfach vor, jedes als separate Zielfunktion modelliertes Ziel in eine einzige Gesamt-Zielfunktion zu integrieren.

So wie die Systemarchitektur bisher dargestellt wurde, ergäbe sich die Lösungsermittlung (LE) aus einem einzigen monolithischen OP. Für die Lösung praktischer Probleme wäre es oft hilfreich, diesen Monolithen zur besseren Übersichtlichkeit in Teil-OPs strukturieren zu können. Abbildung 9 deutet dies auf der linken Seite an: Die gestrichelten Linien zerlegen das gesamte OP in Teil-OPs. Die Linien sind gestrichelt, da im Allg. gewissen Abhängigkeiten zwischen diesen Teil-OPs bestehen, also keine strenge Separierung möglich ist. Diese visuelle, intuitive Darstellung der OP-Strukturierung ist rechts in Abbildung 9 formal vorgenommen worden, entlang evtl. vorhandener Teil-Zielfunktionen sowie der zugehörigen Aspekte der Nebenbedingungen. Die Strukturierung der Nebenbedingungen erfolgt durch Gliederung der beteiligten Systemfunktionen $f_{\text {int }, S}, f_{\text {int }, E}, f_{\text {ext }, E}$. Zu diesem Zweck wird jede dieser Funktion als aus Teil-Funktionen zusammengesetzt de- 


\section{Unternehmensziele}

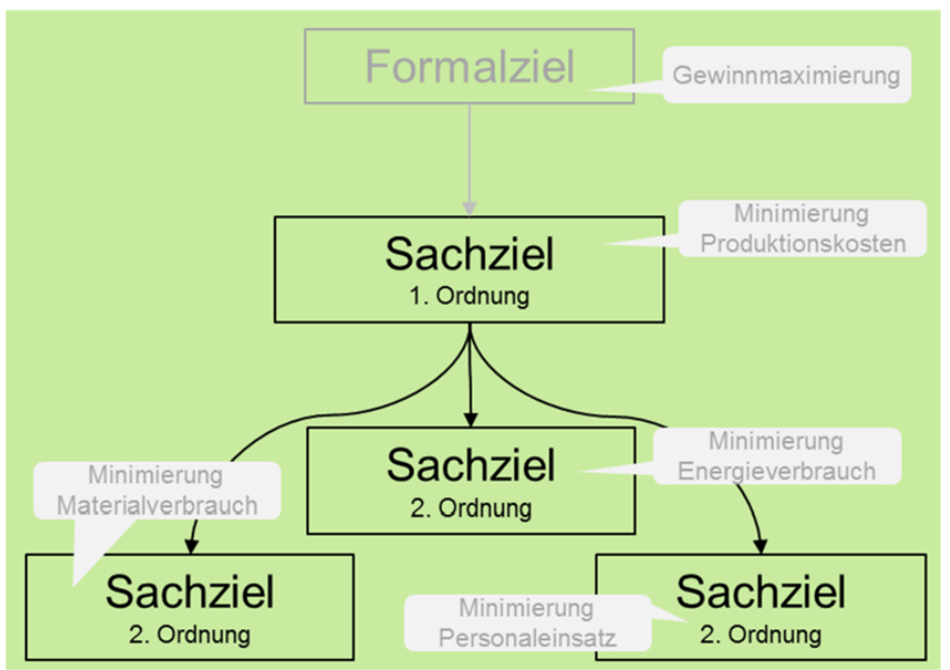

Abb. 10: Exemplarische Zielhierarchie in der Industrie 4.0.

finiert. Jede dieser Teil-Funktionen gehört dann wie dargestellt zu einem Teil-OP. Die Funktionen $\tilde{f}_{\text {int }, S}, \tilde{f}_{\text {int }, E}, \tilde{f}_{\text {ext }, E}$ führen die jeweiligen Teil-Funktionen zu den entsprechenden Systemfunktionen zusammen. Dabei können auch evtl. Abhängigkeiten zwischen den Teilfunktionen ausgedrückt werden für den Fall, dass eine strenge Separierbarkeit nicht möglich ist. Die übrigen Bestandteile der Nebenbedingungen $A A S_{S} \bigvee_{S}$ sowie $A A S_{E} \bigvee_{E}$ werden als allen TeilOPs zugehörig aufgefasst. Natürlich wäre auch hier eine Strukturierung in Form von Teilmengen möglich.

Für die Skill-Zuordnung zwischen in der Lösungsermittlung errechneten Anforderungen an die Problemlösung und verfügbaren Skills zur Lösungsumsetzung ist die Frage zu klären, wie diese Anforderungen entsprechenden Skills (Fähigkeitszusicherungen) zugeordnet werden können. Der Vorschlag ist hier, dass dies durch eine passende Interpretation ${ }^{10}$ der Entscheidungsvariablen aus dem OP erfolgt. Ein simples Beispiel soll das verdeutlichen: In dem OP der Lösungsermittlung könnte es bspw. eine Entscheidungsvariable $n_{\text {ProdA }}$ geben. Diese wird durch die Lösung des OPs z. B. mit $n_{\text {ProdA }}=5$ belegt. Die Interpretation der Variable $n_{\text {ProdA }}$ könnte nun so gewählt sein, dass dies als Anforderung zu verstehen ist, 5 Einheiten von Produkt A produzieren zu müssen, um eine Zielfunktionswerterhöhung zu erreichen. Entsprechend dieser Interpretation ist also lokal oder global nach einem Skill zu suchen, welche die Anzahl produzierter Einheiten von Produkt A beeinflussen kann. Anders ausgedrückt: Es ist nach der Fä-

10 Der Begriff der Interpretation stammt aus der mathematischen Modelltheorie und ist bspw. in [21] definiert. higkeit zu suchen, Einheiten von Produkt A herstellen zu können.

Zuletzt sei noch auf den Inhalt der Zielstellung(en) einer AAS - ausgedrückt durch die Zielfunktion - hingewiesen. Ein allgemeines Modell hat dem AAS-Entwickler keine Vorgaben bezüglich des beabsichtigten AAS-Verhaltens $\mathrm{zu}$ machen. Eine gängige Strukturierung von Unternehmenszielen in einer Zielhierarchie sieht die Unterteilung in Formal- und Sachziele vor [22] wie in Abbildung 10 dargestellt. Das Formalziel, d. h. die grundlegende Motivation hinter dem Einsatz von AASs in der Industrie, dürfte immer die Gewinnmaximierung des Unternehmens sein, das die AAS betreibt. Um dies zu erreichen, sind konkrete umsetzbare Sachziele zu operationalisieren, die dem Zweck der Gewinnmaximierung dienen. Derer kann es innerhalb einer Zielhierarchie mehrere geben und die Sachziele können ihrerseits wieder zu konkreteren Sachzielen verfeinert werden (s. Abbildung 10). Es hängt vom konkreten Verwendungskontext einer AAS ab, was diese Sachziele sind und auf welcher Ebene einer solche Zielhierarchie sich die durch den Entwickler modellierte Zielfunktion einer AAS einordnen lässt.

\section{Kritische Einordnung des Vorschlags}

An die vorgestellte Architektur proaktiver AASs wurden eingangs einige Anforderungen gestellt. 
Die Anforderung der Allgemeingültigkeit wird durch Bezugnahme auf generelle und in den entsprechenden Fachdisziplinen etablierte kognitionswissenschaftliche Konzepte adressiert. Vor allem, aber nicht ausschließlich, wurde hierbei das Modell der Phasen des Problemlösens herangezogen, das sich leicht in der Architektur wiederfinden lässt und somit deren Plausibilität erhöht. Aufgrund der Vielfalt und Komplexität möglicher I4.0-Szenarien wird hier davon ausgegangen, dass sich diese Allgemeingültigkeit nicht endgültig formal ableiten lässt, sondern - wie in vorliegender Arbeit angestrebt zunächst plausibilisiert wird. Daran anschließend werden exemplarisch praxisrelevante Szenarien mithilfe der vorgeschlagenen Architektur umgesetzt, um zusätzlich eine empirische Validierung vorzunehmen. Dies soll sowohl in qualitativer als auch quantitativer Hinsicht erfolgen. Erkenntnisse dieser Evaluationsphase fließen nötigenfalls in eine Anpassung oder Erweiterung der Architektur ein. Die dafür herangezogenen Anwendungsfälle werden so gewählt, dass deren Umsetzung ein hohes Maß an Autonomie der beteiligten AASs erfordert.

Die Architektur besteht aus hinsichtlich Zweck, Funktionsweise und gegenseitiger Wechselwirkungen definierten Komponenten. Ein zentraler Bestandteil dieser Definition ist das formal definierte anwendungsunabhängige Konzept für die Modellierung von Verhalten proaktiver AASs. Diese Formalität soll eine systematische und somit nachvollziehbare Spezifikation sowie Implementierung von AASs ermöglichen. Formalität ist insbesondere im Umfeld der deutschen I4.0-Aktivitäten zurzeit eine kaum berücksichtigte Anforderung.

Die Anforderung der Anwendbarkeit für offene Umgebungen wird durch den dynamischen Charakter des zugrundeliegenden Modells des Problemlösens und die Einordnung von Ansätzen zur Behandlung von Unsicherheit adressiert. Dadurch lassen sich AAS-Implementierungen in unzugänglichen, nicht-deterministischen und dynamischen Umgebungen einsetzen. Das volle Potential der Industrie 4.0 wird erst mit der Befähigung von Systemen für den Umgang mit derartigen Umgebungen ausgeschöpft [4].

\section{Spezialisierung des proaktiven Verhaltensmodells zur Zustandsmaschine}

Die Systemarchitektur hat einen recht generischen Charakter, um auf die Problemklasse aller I4.0-Szenarien an- wendbar zu sein und nicht lediglich Einzelprobleme $\mathrm{zu}$ adressieren. Oben wurde als allgemeinste Form für die Repräsentation des proaktiven Verhaltens einer AAS eine spezielle Form für ein OP eingeführt. Der Vorteil einer solchen Verhaltensspezifikation besteht darin, dass der Entwickler das AAS-Verhalten deklarativ angeben kann. Er muss also nicht Lösungswissen „vordenken“ und dieses starr in die AAS implementieren. Stattdessen wird das gewünschte Verhalten formal in Form des angegeben OPs abgebildet und ein geeignet gewählter OP-Solver sorgt dafür, dass in jeder konkreten Problemsituation, in der die AAS agiert, das geforderte Verhalten gezeigt wird. Will man das proaktive Verhalten einer AAS beispielsweise durch eine Zustandsmaschine repräsentieren, so sind durch den Entwickler die konkreten Problemsituationen, in der die AAS später im operativen Betrieb agieren wird, vorherzusehen und die jeweiligen Lösungen für diese Problemsituationen vorzuimplementieren. Dies schränkt den Grad der Autonomie [5] einer so entwickelten AAS ein, da diese somit nicht in der Lage ist, auf durch den Entwickler nicht vorhergesehene Situationen entsprechend des gewünschten Verhaltens zu reagieren. Echte Autonomie entsteht gerade erst dadurch, dass eine AAS kein für konkrete Problemsituationen passendes Lösungswissen in sich trägt, sondern stattdessen ausschließlich Wissen über ihren Zweck - d. h. über Motivation und Ziel - sowie ihre eigenen Handlungsalternativen und ausreichend Umgebungswissen beinhaltet. Genau dies lässt sich mit der vorgestellten OP-Form deklarativ ausdrücken.

Nun wird es jedoch in der Praxis Szenarien geben, in der der erwähnte hohe Grad an Autonomie gar nicht benötigt, gewünscht oder mit vertretbarem Aufwand realisierbar ist. Das ist der Grund dafür, dass Verhaltensspezifikationen ${ }^{11}$ in der SW-Entwicklung oft auch in anderen Formen getätigt werden. Beliebt ist die Verwendung von Zustandsmaschinen (kurz: ZM), die hier exemplarisch und stellvertretend für eine Vielzahl weiterer aus der OPForm ableitbarer Möglichkeiten zur Verhaltensspezifikation betrachtet wird. Es existieren verschiedene Typen von ZMs und entsprechende mathematische Modelle. Beispielsweise lässt sich ein Mealy-Transduktor darstellen als ein 6-Tupel $\left(\Sigma, \Gamma, S, s_{0}, \delta, \omega\right)$ wobei gilt [23]:

- $\quad \Sigma$... Eingabealphabet

- $\quad$.... Ausgabealphabet

- $S$... Zustandsmenge,

- $s_{0} \in S \ldots$ Anfangszustand,

- $\delta \ldots$ Zustandsübergangsfunktion: $\delta: S \times \Sigma \rightarrow S$,

- $\quad \omega$... Ausgabefunktion: $\omega: S \times \Sigma \rightarrow \Gamma$

11 Zur Erinnerung: Wir könnten auch „Algorithmus“ sagen. 


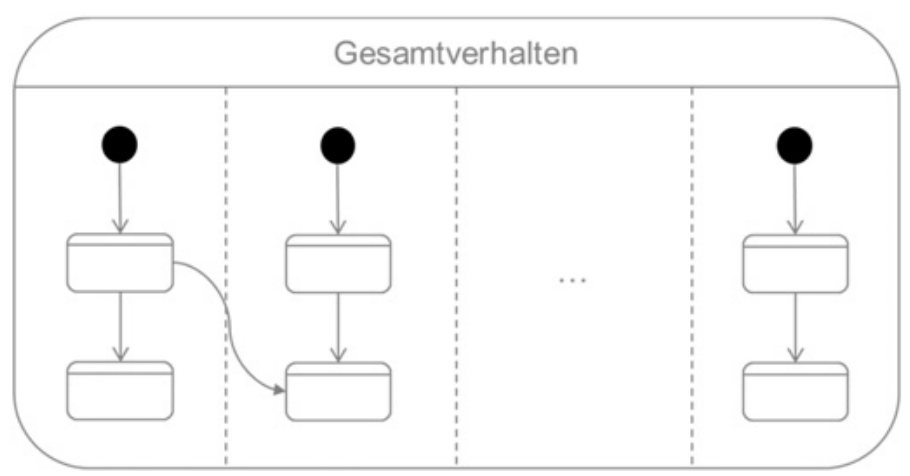

Abb. 11: Strukturierung des Gesamtverhaltens entsprechend der Teil-Zielstellungen einer AAS durch orthogonale Regionen.

Es finden sich zahlreiche Erweiterungen des traditionellen Modells der ZM. So führt bspw. die Modellierungssprache UML die Erweiterungen der hierarchisch verschachtelten Zustände, der orthogonalen Regionen, der Entry/Exit actions oder der internen Transitionen ein [24]. Unter Ausnutzung dieser Möglichkeiten lässt sich das Gesamtverhalten einer AAS schlanker und übersichtlicher spezifizieren. Das ist u. a. dann hilfreich, wenn das proaktive Verhalten einer AAS die Erreichung mehrerer Teil-Ziele anstrebt. Dann kann das Gesamt-Verhalten in entsprechende Teil-Verhalten zerlegt und durch separate orthogonale Regionen repräsentiert werden. Sollten jedoch - wie i. Allg. üblich - Abhängigkeiten zwischen den Teil-Verhalten bestehen, so lässt sich auch dies spezifizieren. Abbildung 11 veranschaulicht das.

Erwähnenswert ist an dieser Stelle, dass eine ZM letztlich nur eine durch den Entwickler vorweggenommene Lösung eines OPs ist, das der oben eingeführten Form entspricht - für eine antizipierte und begrenzte Menge von im operativen Betrieb evtl. auftretenden (Problem-)Situationen. Die Tatsache, dass der Entwickler der AAS das Lösen eins solchen OPs (teilweise) abnimmt, ist ursächlich für den geringeren Grad an Autonomie dieser AAS.

Der Zweck dieses Kapitels besteht nicht darin vorzuschlagen, Verhaltensspezifikationen für eine AAS in jedem Fall in Form eines OPs anzugeben. Die Ausführungen sollen jedoch darauf hinweisen, dass die vielfach verwendeten ZMs ein Spezialfall des hier vorgeschlagenen generischen Modells sind und somit nicht für alle Szenarien geeignet sind und eben auch den Grad der Autonomie einer AAS erheblich beeinträchtigen. Schlägt man für eine grundlegende Systemarchitektur für AASs die Verwendung von ZMs vor, so wird bei genauerer Betrachtung Verwunderung darüber aufkommen, dass solch eine Architektur keinen Platz vorsieht für die Umsetzung von im I4.0-Umfeld viel diskutierten Ideen wie „Motivation“, „Zielorientierung“ oder „Proaktivität“.

\section{Dezentralität durch konsensuale Regeln/Interaktionsmuster}

Das Konzept der Verwaltungsschale soll dezentral organisierte Systeme ermöglichen. Damit Dezentralität überhaupt realisierbar ist, sind einige von jedem Systemteilnehmer zu implementierende Verhaltensregeln notwendig. Denn eine koordinierende zentrale Instanz, die Chaos vermeidet, globale Beschränkungen überwacht oder Wechselwirkungen zwischen Systemteilnehmern beachtet, existiert nicht [25]. An deren Stelle treten die Verhaltensregeln, die gewissermaßen globale „Sinnhaftigkeit“ durch lokale „Sinnhaftigkeit“ ermöglichen sollen. Um noch mal einen Bezug zu den Kognitionswissenschaften herzustellen, sei auf [26] verwiesen, nach der das Sprechen wie auch jede andere Form des zweckgerichteten Handelns Regeln folgt, die im allgemeinen durch (soziales) Training von allen Teilnehmern eines dezentralen Systems, wie z. B. einer Sprachgemeinschaft, beherrscht werden müssen. Dies allein ist demnach notwendig und hinreichend, um Verständigung - oder allgemein sinnhaftes Handeln - zu ermöglichen. Technisch gesehen, würde sich dieses Regelwerk in dem oben angegebenen OP als Teil der Zielfunktion oder der Nebenbedingungen niederschlagen (Abbildung 12).

Ein Beispiel für eine solche Regel wäre eine Beseitigungsstrategie für einen Deadlock-Zustand, in dem sich 2 oder mehr Teilnehmer innerhalb ihres Interaktionsgebarens befinden könnten. Im simpelsten Fall würde diese Strategie schlicht vorschreiben, etwas anders zu machen als bisher, denn das bisherige Verhalten hat ja den Deadlock-Zustand hervorgebracht und aufrechterhalten. Auch der Mensch, als das eindrücklichste Beispiel für ein grundsätzlich dezentral agierendes System, agiert auf diese Weise. Das durch alle Systemteilnehmer (Gesellschaft) zu implementierende Regelwerk sind hierbei gel- 


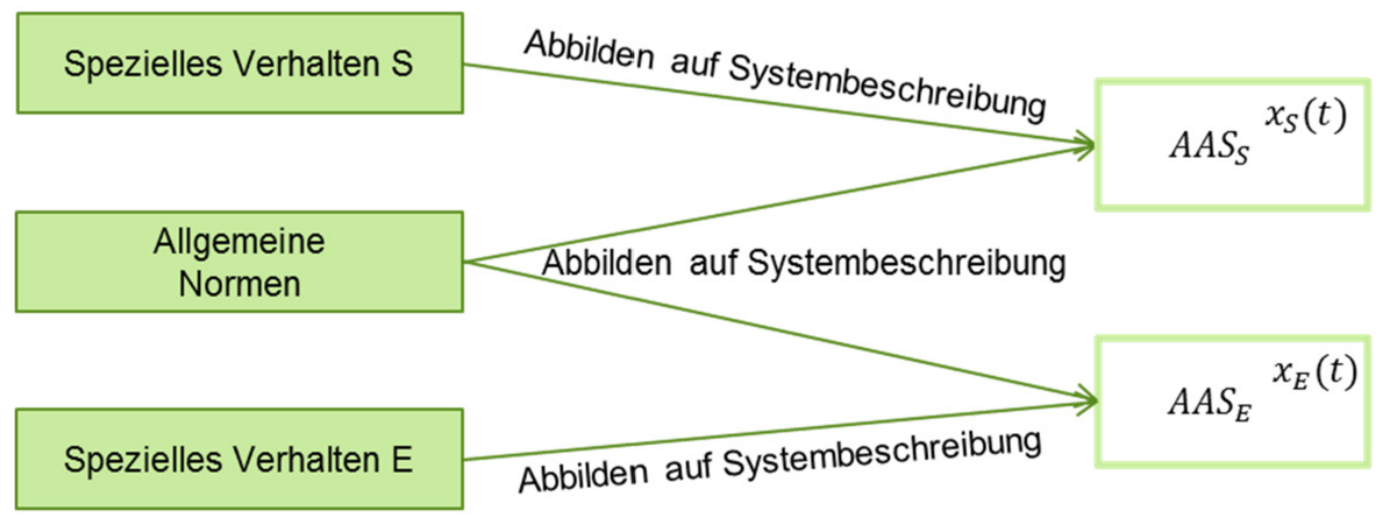

Abb. 12: Regelwerk als Teil der Zielfunktion oder der Nebenbedingungen des OPs.

tende Normen (Gesetze, Konventionen). Dies scheint ein Indikator dafür zu sein, dass die Idee der konsensualen Regeln geeignet ist, um Dezentralität/Autonomie zu realisieren. Es hängt von der Ausgestaltung des Regelwerkes ab, wie zufriedenstellend die so umgesetzten Systeme arbeiten.

\section{Technologische Umsetzung}

Um das vorgestellte Konzept nutzen zu können, werden geeignete Technologien benötigt. Es wird hier der Standpunkt vertreten, dass die Fülle der existenten Technologien schon geeignete Mittel bereitstellt. Eine Neu-Erfindung von Technologien scheint nicht nötig. Anhand der in Abbildung 4 vorgestellten Systemarchitektur lassen sich relevante Technologiefelder ableiten.

Die Umsetzung der Daten-Komponente erfordert Technologien zur Daten-/Informations-/Wissensrepräsentation. Ein Überblick über einige der zahlreichen verfügbaren Technologien hierzu und zugehöriger Werkzeugunterstützung findet sich in $[27,28]$.

Die Umsetzung der Algorithmen-Komponente erfordert Technologien zur algorithmischen Verarbeitung von Daten. Auf deren Sub-Komponenten wurde im Verlauf der Arbeit bereits vereinzelt eingegangen. So existieren zahlreiche Konzepte samt Software-Bibliotheken für die Ausgestaltung der Lern-Komponente. Dasselbe gilt für die innerhalb der LE-Komponente benötigten Mittel zur Realisierung des OP-Solvers. Die Programmierung von SZKomponente und Skill-Komponente erfordert im Allgemeinen jeweils keine besonderen Mittel.

Etwas betrachtungswürdiger sind die beiden Kommunikationsschnittstellen Asset-IT und AAS-IT. Diesbezüglich werden im I4.0-Umfeld aktuell einige Bemühungen unternommen. Dies liegt vermutlich daran, dass sich vordergründig betrachtet an dieser Stelle die Frage der Interoperabilität entscheidet. Dies bezieht sich jedoch nur auf die technische sowie syntaktische Interoperabilität. Bezüglich dieser geht es technologisch betrachtet um die Auswahl eines geeigneten Kommunikationsprotokollstacks. Wie erwähnt, gibt es zahlreiche Alternativen. In dieser Arbeit wurde soweit wie möglich auf typische Internetprotokolle gesetzt. Diese sind weit verbreitet, was die technische und syntaktische Interoperabilität mit bestehenden Systemen fördert. In der Vergangenheit wurden bereits einige in bestimmten Kontexten auftretende Schwächen der klassischen Internetprotokolle identifiziert und diesen mit Anpassungen begegnet. Dazu zählt zum Beispiel die Entwicklung von Websockets, um unumständlich eine bidirektionale Kommunikation zwischen HTTP-Client und - Server zu ermöglichen. Der Eigenschaft der Zustandslosigkeit von HTTP kann mit der Implementierung einer Zustandshaltung begegnet werden - eine für anspruchslose Szenarien geeignete Möglichkeit ist die Verwendung von Cookies. Auch hinsichtlich der Unterstützung von Maßnahmen für die Security, Authentifizierung und Autorisierung gibt es mit Protokollen wie TLS und OAuth Lösungen. Der vorgeschlagene Protokollstack ist in Abbildung 13 links dargestellt. Auf der rechten Seiten dieser Abbildung ist dem ein OPC UA-Stack gegenübergestellt.

OPC UA $[29,30]$ unterstützt verschiedene so genannte Transportprofile. Dies bedeutet, dass OPC UA nicht einen einzigen, sondern einen bezüglich einiger Ebenen des OSI-Modells konfigurierbaren Stack unterstützt, so wie auch bei Internetprotokollen gewisse Konfigurationsmöglichkeiten bestehen (TLS on/off, Websocket on/off usw.). Hier wurde eine Konfiguration gewählt, die dem InternetProtokollstack möglichst ähnlich ist. Dass HTTP im OPC UA-Stack als Transportprotokoll eingestuft wird, ist zwar 


\begin{tabular}{|c|c|c|c|c|}
\hline Application & $\begin{array}{c}\text { HTTP } \\
\text { (HTTP methods) }\end{array}$ & & $\begin{array}{c}\text { OPC UA } \\
\text { (Service Model, Meta Information Model) }\end{array}$ & \\
\hline Presentation & JSON & & JSON & \\
\hline Session & TLS & $\stackrel{\overrightarrow{\widetilde{D}}}{\vec{\nabla}}$ & TLS & 은 \\
\hline Transport & TCP & $-\frac{\overrightarrow{0}}{\overrightarrow{0}}$ & HTTP & 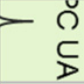 \\
\hline Network & IP & $\frac{\overline{\hat{o}}}{\overline{\bar{D}}}$ & IP & \\
\hline Data Link & Ethernet, TSN & & Ethernet, TSN & \\
\hline Physical & Ethernet & & Ethernet & \\
\hline
\end{tabular}

Abb. 13: Mögliche Protokollstacks für AAS-IT.

ungewöhnlich, wird aber durchaus so gehandhabt, da HTTP hier lediglich als Wrapper um die zu übertragenen Daten genutzt wird, um Firewall-Freundlichkeit zu gewährleisten. Die applikationsschichtspezifischen Eigenschaften von HTTP werden stattdessen durch die applikationsschichtspezifischen Eigenschaften von OPC UA ersetzt. Hierzu zählt das Service-Modell von OPC UA, das anstelle der HTTP-Anfragemethoden steht. Aus der Abbildung lässt sich ein möglicher Wert der Verwendung von OPC UA gegenüber einem reinen Internet-Protokollstack ableiten: OPC UA bietet ein Meta-Informationsmodell auch Address Space Modell genannt. Für den InternetProtokollstack wäre ein solches zunächst zu definieren, was allerdings bei Orientierung an bestehenden objektorientierten Prinzipien einfach möglich sein sollte. Hier ist es denkbar, eine Anpassung des Meta-Modell-Vorschlags aus [14] an das vorgestellte Konzept anzustreben, für den bereits ein JSON-Mapping vorliegt. Ein Unterschied besteht zudem hinsichtlich des Service-Modells. Jedoch bieten sowohl HTTP als auch OPC UA die Möglichkeit, das CRUDKonzept zu nutzen. HTTP bietet dazu u. a. die Anfragemethoden POST, GET, PUT sowie DELETE. OPC UA bietet dazu u. a. die Dienste des Attribute- sowie Node ManagementService Sets. Die Entscheidung zwischen beiden vorgestellten Stacks fiel hier auf den internetprotokoll-basierten Stack, da der OPC UA-bezogene Vorteil der Verfügbarkeit eines Meta-Informationsmodells einfach $z u$ überwinden scheint, dieser Stack aber im Unterschied zu OPC UA bereits sehr etabliert ist und somit keine unnötigen Migrationsanforderungen gestellt werden.

Für beide Stacks gilt, dass die aktuellen Bemühungen zur Echtzeit-Befähigung von Ethernet durch TSN [31] die letzte technologische Lücke hinsichtlich der Kommunika- tionstechnologien für I4.0-Anwendungen schließen könnte.

AASs wurden in dieser Arbeit hinsichtlich ihres Interaktionsverhaltens als zustandsbehaftet, asynchron und nicht-deterministisch charakterisiert. Solche Charakterisierungen finden auch bei Kommunikationsprotokollen Anwendung. So wird HTTP beispielsweise als zustandsloses Protokoll bezeichnet. Man kann diesem Konflikt auf 2 Wegen begegnen. Zum einen bezieht sich die Charakterisierung der AAS auf die Applikationslogik hinsichtlich des Interaktionsverhaltens. Dies bedeutet, dass applikativ zustandsbehaftete AASs problemlos über ein kommunikationstechnisch betrachtet zustandsloses Protokoll kommunizieren können. Die Applikation würde in dem Fall die Zustandshaltung selbst implementieren. Zum anderen ist HTTP wie bereits erwähnt durch Erweiterungen wie Websockets mittlerweile nicht mehr nur in seiner ursprünglichen Charakterisierung - z. B. als zustandsloses Protokoll - verwendbar.

\section{Zusammenfassung \& Ausblick}

Die Arbeit stellt eine Systemarchitektur für proaktive AASs vor. Bei der Ableitung der Systemarchitektur wurden kognitionswissenschaftliche Konzepte berücksichtigt. Ohne eine solche Bezugnahme auf Erkenntnisse aus Disziplinen, die sich fundiert mit Begriffen wie „Intelligenz“, „Autonomie“ oder „Dezentralität“ beschäftigen, ergeben sich schnell Architekturen, die aufgrund zu pragmatischer und einsatzzweck-bezogener Erwägungen an Allgemeingültigkeit verlieren. Dies schränkt deren Anwendbarkeit und Plausibilität ein, sodass kaum ein systematisches Vorge- 
hen zur AAS-Spezifikation und - Entwicklung daraus ableitbar wäre.

Die Arbeit greift bisherige Bestrebungen aus der Plattform Industrie 4.0 auf und entwickelt diese weiter. Erste Ergebnisse der darin angesiedelten Gremien können in die Systemarchitektur eingeordnet werden. So kommt das Meta-Informationsmodell der AAS als Format für die im Datenbereich vorgehaltenen Daten infrage. Auch zeigt sich, dass die Plattform Industrie 4.0 bisher lediglich reaktive AASs adressiert, was die Umsetzung der ursprünglich von denselben Gremien definierten Ziele nicht möglich macht.

Zudem wurde der Beitrag von konsensualen Verhaltensregeln zur erfolgreichen Dezentralisierung von Systemen herausgearbeitet. Diesbezüglich besteht hoher Entwicklungsbedarf, denn derartige Regeln sind noch zu spezifizieren. Abschließend wurde eine exemplarische Betrachtung von geeigneten Realisierungstechnologien zur Umsetzung der Konzepte vorgenommen. Aktuell wird das Konzept auf beispielhafte Szenarien angewendet, um dessen Handhabbarkeit und Validität empirisch zu prüfen.

Die Verfügbarkeit einer solchen Architektur und der Beschreibung der Arbeitsweise der enthaltenen Komponenten und deren Beziehungen ziehen natürlich nicht nach sich, dass I4.0-Szenarien nun stets problemlos implementierbar wären. Es existiert eine Reihe von grundsätzlichen Herausforderungen in der KI-Forschung - auch unabhängig von der Spezialisierung zur Industrie 4.0. Dazu gehört vor allem das Problem der immer steigenden Komplexität der Anwendungsszenarien. Diese Komplexität führt unabhängig von der Tauglichkeit der grundlegenden Konzepte zu immer anspruchsvoller zu entwickelnden AASs. Zu begegnen wäre dem mit Untersuchungen zur Komplexitätsreduktion von Wissensmodellen und Verhaltensspezifikationen. Hier liefert insbesondere die Mathematik wertvolle Beiträge [32]. Zudem ist zu erwarten, dass das in [14] vorgeschlagene und durch die vorgestellte Architektur verwendete Datenmodell zukünftig einigen Erweiterungen und Modifikationen unterliegen wird. Auch dazu wird im Rahmen der Weiterentwicklung des vorgestellten Konzepts beigetragen.

\section{Literatur}

1. Alliance Industrie du Futur, Piano Industria 4.0, Plattform Industrie 4.0 (Hrsg.): The Structure of the Administration Shell: TRILATERAL PERSPECTIVES from France, Italy and Germany International paper, 2018.
2. Turnitsa, C. D.: Extending the Levels of Conceptual Interoperability Model. Proceedings IEEE Summer Computer Simulation Conference, 2005.

3. Reich, J.; Schröder, T.: A reference model for interaction semantics. https://arxiv.org/pdf/1801.04185.pdf, 2017.

4. Plattform Industrie 4.0 (Hrsg.): Aspekte der Forschungsroadmap in den Anwendungsszenarien, 2016.

5. Gronau, N.; Grum, M.; Bender, B: Determining the optimal level of autonomy in cyber-physical production systems. IEEE 14th International Conference on Industrial Informatics (INDIN), 2016.

6. Wooldridge, M.: An Introduction to MultiAgent Systems. John Wiley \& Sons, 2002.

7. Schmidhuber, J.: Formal Theory of Creativity, Fun, and Intrinsic Motivation. IEEE Transactions on Autonomous Mental Development, 2(3), 2010.

8. Ryan, R. M.; Deci, E. L.: Self Determination Theory and the facilitation of intrinsic motivation, social development and well-being. American Psychologist, 55(1), 2000.

9. Allingham, M.: Choice Theory: A Very Short Introduction. Oxford University Press, 2002.

10. Betsch, T.; Funke, J.; Plessner, H.: Denken - Urteilen, Entscheiden, Problemlösen. Springer, 2010.

11. Eysenck, M. W.: Fundamentals of cognition. Psychology Press, 2012.

12. Leonard, D. C.: Learning Theories: A to Z. Greenwood, 2002.

13. Lunze, J.: Regelungstechnik 1: Systemtheoretische Grundlagen, Analyse und Entwurf einschleifiger Regelungen. Springer Vieweg, 2016.

14. Plattform Industrie 4.0, ZVEI (Hrsg.): Details of the Asset Administration Shell-Specification, 2018.

15. Hildebrandt, C.; Scholz, A.; Fay, A.; Dubovy, M.; Schröder, T.; Hadlich, T.; Diedrich, C.; Eck, C.; Wiegand, R.: Semantic Modeling for Collaboration and Cooperation of Systems in the production domain. ETFA, 2017.

16. Martin, J.: Managing the Data Base Environment. Pearson Education, Limited, 1983.

17. Beierle C.; Kern-Isberner, G.: Methoden wissensbasierter Systeme: Grundlagen, Algorithmen, Anwendungen (Computational Intelligence). Springer Vieweg, 2014.

18. VDI/VDE (Hrsg.): VDI/VDE 2193 Blatt 1 - Sprache für 14.0-Komponenten - Struktur von Nachrichten, 2019.

19. von Neumann, J.; Morgenstern, O.: Theory of Games and Economic Behavior. Princeton University Press, 1953.

20. Miettinen, K.: Nonlinear Multiobjective Optimization. Springer, 1999.

21. Marker, D.: Model Theory: An Introduction. Graduate Texts in Mathematics 217, Springer, 2002.

22. Wöhe, G.; Döring, U.; Brösel, G.: Einführung in die Allgemeine Betriebswirtschaftslehre. Vahlen, 2016.

23. Mealy, G. H.: A Method for Synthesizing Sequential Circuits. Bell System Technical Journal, pp. 1045-1079, 1955.

24. Rupp, C.; Queins, S.; Zengler, B.: UML 2 glasklar. Carl Hanser Verlag, 2007.

25. Nwana, H.; Lee, L.; Jennings, N.: Coordination in software agent systems. Part of Fellowship at BT Labs, 1996.

26. Mounce, H. O.: Wittgenstein's Tractatus. An Introduction. Blackwell, 1990.

27. Brachman, R.; Levesque, H.: Knowledge Representation and Reasoning (The Morgan Kaufmann Series in Artificial 
Intelligence). Morgan Kaufmann, 2004.

28. Hangos, K. M.; Lakner, R.; Gerzson, M.: Tools for Knowledge Representation and Reasoning. Intelligent Control Systems, Applied Optimization, vol. 60, Springer, 2004.

29. Mahnke, W.; Leitner, S.-H.; Damm, M.: OPC Unified Architecture. Springer, 2009.

30. OPC Foundation: OPC Unified Architecture Specification - Part 1: Overview and Concepts. 2017.

31. Bruckner, D.; Blair, R.; Stanica, M.-P.; Ademaj, A.; Skeffington, W.; Kutscher, D.; Schriegel, S.; Wilmes, R.; Wachswender, K.; Leurs, L.; Seewald, M.; Hummen, R.; Liu, E.-C.; Ravikumar, S.: OPC UA TSN. A new Solution for Industrial Communication. https://www.br-automation.com/downloads_br_ productcatalogue/assets/1519253860692-en-original-1.0.pdf. Version: Februar 2019.

32. DFG-Graduiertenkolleg Mathematische Komplexitätsreduktion. https://www.mathcore.ovgu.de/. Version: Februar 2019.

33. Kruse, R.; Borgelt, C.; Braune, C.; Mostaghim, S.; Steinbrecher, M.; Klawonn, F.; Moewes, C.: Computational Intelligence: $A$ Methodological Introduction (Texts in Computer Science). Springer, 2016.

34. Sternberg, R. J.; Kaufmann, S. B.: The Cambridge Handbook of Intelligence (Cambridge Handbooks in Psychology). Cambridge University Press, 2011.

\section{Autoreninformationen}

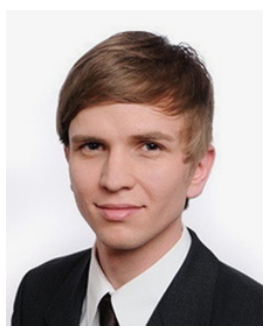

\section{Tizian Schröder}

OVGU Magdeburg, Magdeburg,

Deutschland

tizian.schroeder@ovgu.de

M. Sc. Tizian Schröder (geb. 1991) ist Mitarbeiter am Lehrstuhl Integrierte Automation an der Otto-von-Guericke-Universität (OVGU) Magdeburg. Zuvor studierte er Wirtschaftsingenieurwesen für Elektro- und Informationstechnik mit dem Schwerpunkt Automatisierungstechnik. Seine Hauptarbeitsfelder sind Konzepte und Technologien für semantische Interoperabilität in der digitalen Fabrik.

Tizian Schröder, M. Sc., (b. 1991) now works at the chair for integrated automation at Otto-von-Guericke-Universität (OVGU) in Magdeburg after he studied industrial engineering in the fields of electrical engineering and information technology, focusing on automation technology. His main areas of work are concepts and technologies for semantic interoperability in the digital factory.

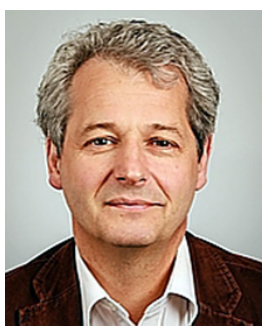

Christian Diedrich

OVGU Magdeburg, Magdeburg, Deutschland

christian.diedrich@ovgu.de

Prof. Dr.-Ing. Christian Diedrich (geb. 1957) hat technische Kybernetik und Automatisierungstechnik studiert und lehrt und forscht an der Otto-von-Guericke-Universität (OVGU) und am Institut für Automation und Kommunikation (ifak) e.V. in Magdeburg. Seine Schwerpunkte liegen auf dem Gebiet der Automation in digital betriebenen Produktionssystemen basierend auf informations- und wissensbasierten Methoden. Dazu gehören vor allem die industrielle Kommunikation, Integrations- sowie Beschreibungsmethoden. Christian Diedrich, Prof. Dr.-Ing., (b. 1957) studied technical cybernetics and automation technology and teaches and researches at the Otto-von-Guericke University (OVGU) and at the Institut für Automation und Kommunikation (ifak) e.V. in Magdeburg. His work focuses on automation in digitally operated production systems based on information- and knowledge-based methods. This includes industrial communication, integration- and description methods. 